\title{
Unbounded Wiener-Hopf Operators and Isomorphic Singular Integral Operators
}

\author{
Domenico P. L. Castrigiano $^{1} \mathbb{C}$
}

Received: 20 September 2020 / Accepted: 30 March 2021 / Published online: 19 April 2021

(c) The Author(s) 2021

\begin{abstract}
Some basics of a theory of unbounded Wiener-Hopf operators (WH) are developed. The alternative is shown that the domain of a WH is either zero or dense. The symbols for non-trivial WH are determined explicitly by an integrability property. WH are characterized by shift invariance. We study in detail WH with rational symbols showing that they are densely defined, closed and have finite dimensional kernels and deficiency spaces. The latter spaces as well as the domains, ranges, spectral and Fredholm points are explicitly determined. Another topic concerns semibounded WH. There is a canonical representation of a semibounded WH using a product of a closable operator and its adjoint. The Friedrichs extension is obtained replacing the operator by its closure. The polar decomposition gives rise to a Hilbert space isomorphism relating a semibounded WH to a singular integral operator of Hilbert transformation type. This remarkable relationship, which allows to transfer results and methods reciprocally, is new also in the thoroughly studied case of bounded WH.
\end{abstract}

Keywords Unbounded Wiener-Hopf operator · Hardy function · Singular integral operator · Hilbert transformation · Rational symbol - Semibounded symbol ·

Fredholm operator

Mathematics Subject Classification Primary 47G10 - 47B35; Secondary 47A53 . 47B25

Communicated by Pierre Portal.

$\bowtie$ Domenico P. L. Castrigiano

castrig@ma.tum.de

1 Technische Universität München Fakultät für Mathematik, Boltzmannstraße 3, 85748 Garching, Germany 


\section{Introduction}

There is an increasing interest in unbounded Toeplitz and Toeplitz-like operators (see 2.2), which will concern also the related Wiener-Hopf operators (WH). So far, results on $\mathrm{WH} W_{\kappa}$ with unbounded symbol $\kappa$ are scarce and probably in the literature there exists no introduction to this subject. So Sect. 2 deals with preliminaries and basics regarding unbounded WH. In particular we are concerned with conditions on the symbol $\kappa$ ensuring that the domain of $W_{\kappa}$ is either the whole space or dense or trivial, and prove that dom $W_{\kappa}$ is either trivial or dense. The symbols with non-trivial $\mathrm{WH}$, which are called proper, are determined by a useful integrability property. A classical result on the eigenvalues of a WH is shown to remain valid in the unbounded case. It implies that non-trivial symmetric WH have no eigenvalues. A further result characterizes WH by their invariance under unilateral shifts. In Sect. 3 WH with rational symbols are studied. They constitute a welcome source of densely defined closed operators with finite index. An explicit description of the domains, ranges, kernels, deficiency spaces, spectral and Fredholm points is given. The remainder of this article deals in Sects. 4 and 5 with densely defined semibounded WH. A semibounded operator $W_{\kappa}$ can be expressed by a product of a closable operator $A$ and its adjoint. Replacing $A$ by its closure one obtains quite naturally a self-adjoint extension $\tilde{W}_{\kappa}$. It is proven to coincide with the Friedrichs extension. Inverting the order of the factors one obtains a singular integral operator $L_{\phi}$ of Hilbert transformation type. For the operators of that type to be non-trivial there is a necessary condition analogous to that for WH. The self-adjoint extensions $\tilde{L}_{\phi}$ and $\tilde{W}_{\kappa}$ are isometric, which follows from the polar decomposition of $\bar{A}$. Actually $\tilde{W}_{\kappa}$ is Hilbert space isomorphic to the reduction of $\tilde{L}_{\phi}$ on $\operatorname{ker}\left(\tilde{L}_{\phi}\right)^{\perp}$, and the spectral representations of $\tilde{L}_{\phi}$ and $\tilde{W}_{\kappa}$ can be achieved in an explicit manner from each other. It is worth noting that this relationship is new also for bounded WH thus contributing to the well-developed theory of the latter. To conclude, this method is illustrated by a non-trivial example diagonalizing Lalescu's operator and the isometrically related singular integral operator. In [24, Sect.3.3] the spectral representations of $W_{1_{[-1,1]}}$ and the finite Hilbert transformation are related to each other by this method.

Notations Let $\mathcal{F}$ denote the Fourier transformation on $L^{2}(\mathbb{R})$. For measurable $E \subset \mathbb{R}$ introduce the projection $P_{E}: L^{2}(\mathbb{R}) \rightarrow L^{2}(E),\left(P_{E} f\right)(x):=f(x)$, i.e., $P_{E} f=\left.f\right|_{E}$. (For convenience define $L^{2}(E)=\{0\}, P_{E}=0$ if $E=\emptyset$.) Its adjoint $P_{E}^{*}: L^{2}(E) \rightarrow$ $L^{2}(\mathbb{R})$ is the injection $\left(P_{E}^{*} f\right)(x)=f(x)$ for $x \in E$ and $=0$ otherwise. Note

$$
P_{E} P_{E}^{*}=I_{L^{2}(E)}, \quad P_{E}^{*} P_{E}=M\left(1_{E}\right)
$$

with $M\left(1_{E}\right)$ the multiplication by the indicator function $1_{E}$ for $E$. Put $\left.\mathbb{R}_{+}:=\right] 0, \infty[$ and $P_{+}:=P_{\mathbb{R}_{+}}$. Analogously define $P_{-}$. - We call $E$ proper if neither $E$ nor the complement $\mathbb{R} \backslash E$ is a null set.

Throughout let $\kappa: \mathbb{R} \rightarrow \mathbb{C}$ denote a measurable function and $M(\kappa)$ the multiplication operator by $\kappa$ in $L^{2}(\mathbb{R})$ with dense domain $\left\{f \in L^{2}(\mathbb{R}): \kappa f \in L^{2}(\mathbb{R})\right\}$. Recall that $M(\kappa)$ is normal satisfying $M(\kappa)^{*}=M(\bar{\kappa})$ and $M(\bar{\kappa}) M(\kappa)=M\left(|\kappa|^{2}\right)$. 
Moreover, $M(\kappa)$ is self-adjoint if and only if $\kappa$ is almost real, and $M(\kappa)$ is nonnegative if and only if $\kappa \geq 0$ a.e.

Definition 1.1 The operator in $L^{2}\left(\mathbb{R}_{+}\right)$

$$
W_{\kappa}:=P_{+} \mathcal{F} M(\kappa) \mathcal{F}^{-1} P_{+}^{*}
$$

is called the Wiener-Hopf operator (WH) with symbol $\kappa$. Occasionally we write $W(\kappa)$ instead of $W_{\kappa}$. Clearly, $W_{\kappa}=W_{\kappa^{\prime}}$ if $\kappa=\kappa^{\prime}$ a.e. Often we shall refer to this tacitly. The symbol $\kappa$ is called proper if dom $W_{\kappa} \neq\{0\}$.

The theory of WH with bounded symbol is well developed. We content ourselves to refer here to the book [1, Chapter 9] and to mention the origins [2]. Obviously in case of a bounded symbol the operators $W_{\kappa}$ are bounded with dom $W_{\kappa}=L^{2}\left(\mathbb{R}_{+}\right)$and adjoint $W_{\kappa}^{*}=W_{\bar{\kappa}}$. $W_{\kappa}$ is the convolution on the real half line with kernel $k$, i.e.,

$$
\left(W_{\kappa} g\right)(x)=\int_{0}^{\infty} k(x-y) g(x) \mathrm{d} y
$$

if $\kappa \in L^{\infty} \cap L^{2}$ and $k:=(2 \pi)^{-1 / 2} \mathcal{F} \kappa$, or if $\kappa=\int \mathrm{e}^{\mathrm{i}(\cdot) y} k(y) \mathrm{d} y$ for $k \in L^{1}(\mathbb{R})$. For the case of integrable kernel there is the rather exhaustive theory by M. G. Krein [3]. Generally, the tempered distribution $k:=(2 \pi)^{-1 / 2} \mathcal{F} \kappa$, where $\kappa \in L^{\infty}(\mathbb{R})$ is considered as a regular tempered distribution, satisfies $\mathcal{F} M(\kappa) \mathcal{F}^{-1} u=k \star u$ for every Schwartz function $u$ in the distributional sense (e.g. [4, Theorem IX.4]). For instance the kernel for $W_{-\operatorname{sgn}}$ is the tempered distribution $k(x)=-\frac{1}{x}$ or that for $W_{-\tanh }$ equals $k(x)=(2 \mathrm{i} \sinh (\pi x / 2))^{-1}$. In the literature the generalizations of WH stay mostly within the realm of bounded operators. One deals with the traces (compressions) of bounded bijective operators in a Banach space on a closed subspace [5]. The results concern the solvability of the associated Wiener-Hopf equations.

\section{Unbounded Wiener-Hopf Operators}

As put it by [6] results on unbounded WH are practically inexistent. Indeed they are scarce. See [7, 1.3] for some notes. An important result is due to M. Rosenblum [8], [9], obtained for Toeplitz operators and hence valid for the Hilbert space isomorphic WH $W_{\kappa}$ (see 2.2). So in the case that the symbol $\kappa$ is real bounded below not almost constant and $\left(1+x^{2}\right)^{-1} \kappa$ is integrable, [9] furnishes the spectral representation of the extension $\tilde{W}_{\kappa}$, which is shown to be the Friedrichs extension $4.3,4.4$ and which by [9] is absolutely continuous. - There are investigations on unbounded general WH dealing with conditions for their invertibility [10]. - In [6] real bounded below Wiener-Hopf quadratic forms from distributional kernels $k$ are considered, and it is shown that such a form determines a WH if and only if the form is closable or, equivalently, if and only if $\sqrt{2 \pi} k$ is the Fourier transform of a locally integrable bounded below function $\kappa$ with integrable $\left(1+x^{2}\right)^{-n} \kappa$ for some $n \in \mathbb{N}$. Clearly $\kappa$ is the symbol and dom $W_{\kappa} \supset C_{c}^{\infty}\left(\mathbb{R}_{+}\right)$holds. See further [7]. - Furthermore, the 
methods applied for the study of unbounded analytic Toeplitz operators [11] can also produce results on unbounded WH, see 2.10, 4.2, 4.3(c). One deals with $M_{+}(\kappa)(2.2)$, which is canonically Hilbert space isomorphic to $W_{\kappa}$. The former is the trace on the Hardy space $H_{+}$of the multiplication operator by $\kappa$. Rather recently, as indicated to us by the reviewer, in [12] one finds a detailed study of the kernel of $M_{+}(\kappa)$.

\section{Preliminaries}

Starting the preliminary remarks and anticipating briefly some results on $\mathrm{WH}$, note first that dom $W_{\kappa}=\operatorname{dom} M(\kappa) \mathcal{F}^{-1} P_{+}^{*}$. So $\kappa$ is proper if and only if $\kappa h$ is squareintegrable for some Hardy function $2.1 \mathrm{~h} \in \operatorname{ran}\left(\mathcal{F}^{-1} P_{+}^{*}\right)$. Clearly dom $W_{\kappa} \subset \operatorname{dom} W_{\kappa^{\prime}}$ if $\left|\kappa^{\prime}\right| \leq|\kappa|$. Moreover, dom $W_{\kappa}=\operatorname{dom} W_{\bar{\kappa}}=\operatorname{dom} W_{|\kappa|}=\operatorname{dom} W(1+|\kappa|)$. Note $\left\langle g^{\prime}, W_{\kappa} g\right\rangle=\left\langle W_{\bar{\kappa}} g^{\prime}, g\right\rangle$ for $g, g^{\prime} \in \operatorname{dom} W_{\kappa}$. Hence, if $W_{\kappa}$ is densely defined then $W_{\kappa} \subset W_{\bar{\kappa}}^{*}$, whence $W_{\kappa}$ is closable and dom $W_{\kappa} \subset \operatorname{dom} W_{\kappa}^{*}$ holds. But unbounded WH may and may not be closed 3.2,3.5.

If the symbol $\kappa$ is unbounded, then dom $W_{\kappa} \neq L^{2}\left(\mathbb{R}_{+}\right) 2.3$. The alternative holds that either dom $W_{\kappa}$ is trivial or dom $W_{\kappa}$ is dense. In other words, as shown in 2.10, if $\kappa$ is proper, then $W_{\kappa}$ is densely defined. In 2.6 and 2.8 explicit characterizations of proper symbols are given. There is also the useful criterion in 2.4 for $\kappa$ to be proper. So proper symbols may have polynomial growth and countably many singularities with integrable logarithm like as $\exp |x|^{\alpha},-1<\alpha<0$. It is easy to give examples of non-proper symbols 2.9 .

If $W_{\kappa}$ is densely defined then $W_{\kappa}$ is symmetric, i.e., $W_{\kappa} \subset W_{\kappa}^{*}$, if and only if $\kappa$ is almost real 2.14. If $W_{\kappa}$ is densely defined symmetric, then $W_{\kappa}$ is bounded below if and only if $\kappa$ is essentially bounded below 2.15. Recall that the numerical range $\left\{\left\langle g, W_{\kappa} g\right\rangle: g \in \operatorname{dom} W_{\kappa},\|g\|=1\right\}$ of $W_{\kappa}$ is convex. (Indeed, the numerical range of every operator in Hilbert space is convex [13].) It is determined in 2.15, 2.16.

Densely defined symmetric $\mathrm{WH}$, which are not a multiple of $I$, have no eigenvalues 2.11. As already mentioned, if $W_{\kappa}$ is bounded below not a multiple of $I$ with integrable $\left(1+x^{2}\right)^{-1} \kappa$, it follows from [9] that the Friedrichs extension $\tilde{W}_{\kappa} 4.4$ of $W_{\kappa}$ is even absolutely continuous. Section 4 is concerned with the general case of densely defined symmetric semibounded WH and their Friedrichs extensions.

If $\kappa$ is proper real and even (i.e. $\kappa(-x)=\kappa(x))$ then $W_{\kappa}$ is densely defined symmetric and has a self-adjoint extension. This holds true since $L^{2}\left(\mathbb{R}_{+}\right) \rightarrow L^{2}\left(\mathbb{R}_{+}\right)$, $g \mapsto \bar{g}$ is a conjugation, which leaves dom $W_{\kappa}$ invariant and satisfies $W_{\kappa} \bar{g}=\overline{W_{\kappa} g}$ (see [4, Theorem X.3]). If $\kappa$ is odd instead of even then in general $W_{\kappa}$ has no selfadjoint extension. Examples are furnished by real rational symbols as e.g. $\kappa(x)=x$. In 3.4 an explicit description of the deficiency spaces of $W_{\kappa}$ for real rational $\kappa$ are given yielding further examples of densely defined symmetric WH with self-adjoint extensions.

Hardy spaces 2.1 Recall the Hardy spaces $H_{ \pm}:=\operatorname{ran}\left(\mathcal{F}^{-1} P_{ \pm}^{*}\right)$. Obviously $L^{2}(\mathbb{R})=$ $H_{+}(1) H_{-}$and $h \in H_{+} \Leftrightarrow \bar{h} \in H_{-}$as well as $h \in H_{+} \Leftrightarrow \check{h} \in H_{-}$with $\breve{h}(x):=h(-x)$. We tacitly refer to the well-known Paley-Wiener Theorem characterizing the Fourier transforms of $L^{2}$-functions vanishing on a half-axis, see e.g. [14, Theorem 95]. In particular $h \in H_{+}$if and only if there is a $\phi$ holomorphic on the upper half-plane such 
that its partial maps $\phi_{y}(x):=\phi(x+\mathrm{i} y)$ for $y>0$ satisfy $\phi_{y} \in L^{2}(\mathbb{R}),\left\{\left\|\phi_{y}\right\|: y>0\right\}$ bounded, and $\phi_{y} \rightarrow h$ for $y \rightarrow 0$ in the mean and pointwise a.e. Actually $\phi$ converges to $h$ non-tangentially a.e., and $\left\|\phi_{y}\right\| \uparrow\|h\|$ for $y \downarrow 0$ (see e.g. [15, III 3.3, II 2.6]). Moreover, every $h \in H_{ \pm} \backslash\{0\}$ vanishes only on a null set. Indeed, according to a LuzinPrivalov Theorem [16, IV 2.5] a meromorphic function on the upper or lower half-plane which takes non-tangential boundary value zero on a set of positive Lebesgue measure is zero. The former property is also an immediate consequence of the following result on the modulus of a Hardy function. Let $f \in L^{2}(\mathbb{R}) \backslash\{0\}$ :

$$
\text { There ish } \in H_{+} \text {satisfying }|h|=|f| \text { if and only if } \frac{\ln |f|}{1+x^{2}} \in L^{1}(\mathbb{R})
$$

One proves (2.1) using the outer function with prescribed modulus on the torus ( [17, Chap.3, Def. 1.1 and Prop.3.2]) and the Hilbert space isomorphism $\Gamma$ in 2.2. Recall also [18, Theorem XII ] that $\ln |h| /\left(1+x^{2}\right) \in L^{1}$ if $h \in H_{+}$.

Let $M_{+}(\kappa)$ denote the trace of $M(\kappa)$ on $H_{+}$, i.e.

$$
M_{+}(\kappa)=P_{H_{+}} M(\kappa) P_{H_{+}}^{*}
$$

with $P_{H_{+}}: L^{2}(\mathbb{R}) \rightarrow H_{+}$the orthogonal projection. Note that $P_{H_{+}} \mathcal{F}^{-1} P_{+}^{*}$ : $L^{2}\left(\mathbb{R}_{+}\right) \rightarrow H_{+}$is a Hilbert space isomorphism with its inverse $P_{+} \mathcal{F} P_{H_{+}}^{*}$, by which $W_{\kappa}$ is Hilbert space isomorphic to $M_{+}(\kappa)$. Often it is convenient to deal with $M_{+}(\kappa)$ in place of $W_{\kappa}$. Note that $M_{+}(\kappa)$ extends the multiplication operator in $H_{+}$

$$
M\left(\kappa, H_{+}\right) h:=\kappa h \text { with } \operatorname{dom} M\left(\kappa, H_{+}\right)=\left\{h \in H_{+}: \kappa h \in H_{+}\right\}
$$

The latter is Hilbert space isomorphic to its counterpart the so-called analytic Toeplitz operator with symbol $\omega:=\kappa \circ C^{-1}$, see 2.2. $M_{+}(\kappa)$ and $M\left(\kappa, H_{+}\right)$coincide for rational symbols $\kappa$ holomorphic in the upper half-plane 3.1(c), or more general, if $\kappa$ is the nontangential limit of an outer function on the upper half-plane. Indeed, in this case $\frac{\ln |\kappa|}{1+x^{2}}$ is integrable, whence $\kappa$ is proper by 2.8 since $\ln |1+| \kappa|| \leq \ln 2+\ln |\kappa|$, and $[12,3.3]$ applies. We do not put forward this topic.

Finally $H_{+}^{\infty}$ is the set of all measurable bounded $\alpha: \mathbb{R} \rightarrow \mathbb{C}$ such that there is a bounded holomorphic $A$ on the upper half-plane with the partial maps $A_{y} \rightarrow \alpha$ for $y \rightarrow 0$ pointwise a.e. Actually $A$ converges to $\alpha$ non-tangentially a.e. (see e.g. [15, III 3.3, II 2.6]). Clearly, $H_{+}^{\infty} H_{+} \subset H_{+}$.

Remark on Toeplitz operators 2.2 Let the torus $\mathbb{T}$ be endowed with the normalized Lebesgue measure. The Hardy space $H^{2}(\mathbb{T})$ is the subspace of $L^{2}(\mathbb{T})$ with orthonormal basis $e_{n}(w):=w^{n}, n \in \mathbb{N}_{0}$. Given a measurable $\omega: \mathbb{T} \rightarrow \mathbb{C}$ then, quite analogous to (2.2), the Toeplitz operator $T_{\omega}$ with symbol $\omega$ is defined by

$$
T_{\omega}:=P_{H^{2}(\mathbb{T})} M(\omega) P_{H^{2}(\mathbb{T})}^{*}
$$


Let $\Gamma: H^{2}(\mathbb{T}) \rightarrow H_{+},(\Gamma u)(x):=\frac{\mathrm{i}}{\sqrt{\pi}(x+\mathrm{i})} u(C(x))$ be the Hilbert space isomorphism based on the Cayley transformation $C(x):=\frac{x-\mathrm{i}}{x+\mathrm{i}}$. One has

$$
T_{\omega}=\Gamma^{-1} M_{+}(\omega \circ C) \Gamma
$$

Obviously by this well-known relationship (see e.g. [1, 9.5(e)], [24, 3.3.2(13)]) results and methods regarding Toeplitz operators may be transferred for the study of WH and vice versa. For unbounded Toeplitz and Toeplitz-like operators see: [8], [9] for a spectral theory of Toeplitz operators with bounded below integrable symbols, [7] for closable quadratic forms for semibounded Toeplitz operators, [11] for analytic Toeplitz operators, [19] for Toeplitz-like operators with rational symbols.

\section{Results}

As to 2.3 one recalls that in general an everywhere defined linear operator in a Hilbert space need not be bounded.

Proposition 2.3 dom $W_{\kappa}=L^{2}\left(\mathbb{R}_{+}\right) \Rightarrow \kappa$ is bounded $\Rightarrow \operatorname{dom} W_{\kappa}=L^{2}\left(\mathbb{R}_{+}\right)$and $W_{\kappa}$ is bounded.

Proof The second implication is obvious. As to the first one, assume that $\kappa$ is not bounded. Then there exists $f \in L^{2}(\mathbb{R})$ with $\kappa f \notin L^{2}(\mathbb{R})$. Write $f=h_{+}+h_{-}$with $h_{ \pm} \in H_{ \pm}$. As dom $M_{+}(\kappa)=H_{+}$one has $\kappa h_{+} \in L^{2}(\mathbb{R})$, and hence $\kappa h_{-} \notin L^{2}(\mathbb{R})$. Similarly, $\kappa \bar{f} \notin L^{2}(\mathbb{R}), \bar{f}=\bar{h}_{-}+\bar{h}_{+}$with $\bar{h}_{\mp} \in H_{ \pm}$, and $\kappa \bar{h}_{-} \in L^{2}(\mathbb{R})$. This contradicts $\kappa h_{-} \notin L^{2}(\mathbb{R})$.

There follows a first result on the domain of a WH which applies for instance to rational symbols.

Lemma 2.4 If $\kappa$ q $s \in L^{2}(\mathbb{R})$, where $q$ is a polynomial and $s$ is the inverse Fourier transform of a Schwartz function with support in $\left[0, \infty\left[\right.\right.$, then $q s \in \operatorname{dom} W_{\kappa}$ and $\kappa$ is proper. -Suppose that $\kappa q s \in L^{2}(\mathbb{R})$ for a polynomial $q$ with only real zeros and for every Schwartz function $s$. Then $\operatorname{dom} W_{\kappa} \supset\left\{q\left(\mathrm{i} \frac{\mathrm{d}}{\mathrm{d} x}\right) \phi: \phi \in C_{c}^{\infty}\left(\mathbb{R}_{+}\right)\right\}$.

Proof As to the first claim note that $s$ is a Schwartz function in $H_{+}$, whence $q s \in H_{+}$ by 3.1(c).

Now let $\mathrm{D}$ denote the differential operator $\mathrm{i} \frac{\mathrm{d}}{\mathrm{d} x}$ and let $u$ be any Schwartz function with support in $\mathbb{R}_{+}$. Then $q(\mathrm{D}) u$ is still such a function, and $\mathcal{F}^{-1} u, \mathcal{F}^{-1}(q(\mathrm{D}) u)=$ $q \mathcal{F}^{-1} u$ are Schwartz functions in $H_{+}$. Hence it remains to show that $\{q(\mathrm{D}) \phi: \phi \in \mathcal{D}\}$ is dense in $L^{2}\left(\mathbb{R}_{+}\right)$, where $\mathcal{D}$ denotes the space of test functions $C_{c}^{\infty}\left(\mathbb{R}_{+}\right)$. Assume $g \in L^{2}\left(\mathbb{R}_{+}\right)$with $g \perp q(\mathrm{D}) \phi$, i.e., $\int_{\mathbb{R}_{+}} \bar{g} q(\mathrm{D}) \phi \mathrm{d} x=0$. The claim is $g=0$.

Indeed, regarding $\bar{g}$ as a regular distribution in $\mathcal{D}^{\prime}$, one has $\bar{g}(q(\mathrm{D}) \phi)=$ $q(-\mathrm{D}) \bar{g}(\phi)=0$ for all test functions $\phi$, whence $q(-\mathrm{D}) \bar{g}=0$. Thus $\bar{g}$ is a solution of the differential equation $q(-\mathrm{D}) F=0$ for $F \in \mathcal{D}^{\prime}$. As known all its solutions are regular. Hence $\bar{g} \in T$, where $T$ denotes the space of linear combinations of functions on $\mathbb{R}_{+}$ of the kind $x \rightarrow x^{k} \mathrm{e}^{\mathrm{i} \lambda x}, k \in \mathbb{N}_{0}$ and $\lambda \in \mathbb{R}$. Then $G \in T$ for $G(x):=\int_{0}^{x}|g(t)|^{2} \mathrm{~d} t$. 
One has $G(x) \rightarrow\|g\|^{2}<\infty$ for $x \rightarrow \infty$. Write $G=\sum_{k=0}^{n} x^{k} A_{k}$ with $A_{k}$ a linear combination of periodic functions $\mathrm{e}^{\mathrm{i} \lambda x}$. Then $A_{n}(x) \rightarrow 0$ for $x \rightarrow \infty$. Since $A_{n}$ is almost periodic this implies $A_{n}=0$. The result follows.

The following invariance $H_{+}^{\infty} \operatorname{dom} M_{+}(\kappa) \subset \operatorname{dom} M_{+}(\kappa)$ is essential for the density of dom $W_{\kappa}$ in 2.10 .

Lemma 2.5 (a) $W_{\alpha}\left(\operatorname{dom} W_{\kappa}\right) \subset \operatorname{dom} W_{\kappa} \forall \alpha \in H_{+}^{\infty}$, equivalently $\alpha h \in \operatorname{dom} M_{+}(\kappa)$ $\forall \alpha \in H_{+}^{\infty}, h \in \operatorname{dom} M_{+}(\kappa)$.

(b) $\forall \alpha \in H_{+}^{\infty}$ one has (1) $W_{\kappa} W_{\alpha}=W_{\alpha \kappa}$ and (2) $W_{\alpha}^{*} W_{\kappa} \subset W_{\bar{\alpha} \kappa}$.

(c) For $b \geq 0$ let $S_{b}: L^{2}(\mathbb{R}) \rightarrow L^{2}(\mathbb{R})$ be the unilateral translation $S_{b} g(x):=$ $g(x-b)$ if $x>b$ and $:=0$ otherwise. One has the translational invariance $W_{\kappa}=S_{b}^{*} W_{\kappa} S_{b}$.

Proof (a) Let $h \in \operatorname{dom} M_{+}(\kappa)$. The claim is $M_{+}(\alpha) h \in \operatorname{dom} M_{+}(\kappa)$. Now $M_{+}(\alpha) h=\alpha h$ since obviously $\alpha h \in H_{+}$. Moreover $\kappa \alpha h \in L^{2}(\mathbb{R})$, whence the claim.

(b) For (1) note $h \in \operatorname{dom}\left(M_{+}(\kappa) M_{+}(\alpha)\right) \Leftrightarrow h \in H_{+}, P_{H_{+}}(\alpha h) \in \operatorname{dom} M_{+}(\kappa)$. Since $\alpha h \in H_{+}$, the latter is equivalent to $\alpha h \in \operatorname{dom} M_{+}(\kappa) \Leftrightarrow \kappa \alpha h \in$ $L^{2}(\mathbb{R}) \Leftrightarrow h \in \operatorname{dom} M_{+}(\alpha \kappa)$. - As to $(2), M_{+}^{*}(\alpha)=M_{+}(\bar{\alpha}), \operatorname{dom} M_{+}(\kappa)=$ $\operatorname{dom}\left(M_{+}^{*}(\alpha) M_{+}(\kappa)\right)$, and $P_{H_{+}}^{*} P_{H_{+}}=I-P_{H_{-}}^{*} P_{H_{-}}$for $P_{H_{-}}:=I-P_{H_{+}}$. So for $h \in \operatorname{dom} M_{+}(\kappa)$ one has $M_{+}^{*}(\alpha) M_{+}(\kappa) h=M_{+}(\bar{\alpha}) P_{H_{+}}(\kappa h)=$ $P_{H_{+}} M(\bar{\alpha}) P_{H_{+}}^{*} P_{H_{+}}(\kappa h)=P_{H_{+}}(\bar{\alpha} \kappa h)-P_{H_{+}}\left(\bar{\alpha} P_{H_{-}}^{*} P_{H_{-}}(\kappa h)\right)=M_{+}(\bar{\alpha} \kappa) h-0$, since $\bar{\alpha} P_{H_{-}}^{*} P_{H_{-}}(\kappa h) \in H_{-}$.

(c) Check $S_{b}=W\left(\mathrm{e}^{\mathrm{i} b(\cdot)}\right)$, dom $W\left(\mathrm{e}^{-\mathrm{i} b(\cdot)} \kappa\right)=\operatorname{dom} W_{\kappa}$ and apply the foregoing results.

We turn to characterizations of proper symbols $\kappa$, as in particular by the condition of the integrability of $\ln (1+|\kappa|) /\left(1+x^{2}\right)$ in 2.8 .

Proposition 2.6 Let $p \in] 0, \infty]$. Let $j$ denote a real-valued function such that $\frac{j}{1+x^{2}}$ is integrable. Then

$$
\kappa \text { proper } \Leftrightarrow \kappa \mathrm{e}^{j} \in L^{p}(\mathbb{R}) \text { for some } j
$$

Proof Here we prove the case $p=2$ and the implication $\Leftarrow$ for $p=\infty$ and $p=1$. The remainder is shown in the proof of 2.7.

$\kappa$ being proper there is $h \in \operatorname{dom} M_{+}(\kappa) \backslash\{0\}$. Then $\kappa h \in L^{2}(\mathbb{R})$, whence $\kappa \mathrm{e}^{\ln |h|} \in$ $L^{2}(\mathbb{R})$. By $(2.1), \ln |h| /\left(1+x^{2}\right)$ is integrable. For the converse implication put $j^{\prime}:=$ $1_{\{j \leq 0\}} j-|x|^{1 / 2}$. Then $j^{\prime} /\left(1+x^{2}\right)$ is integrable, $\mathrm{e}^{j^{\prime}}$ is square-integrable. By (2.1) there is $h \in H_{+}$with $|h|=\mathrm{e}^{j^{\prime}}$. Moreover, $\kappa \mathrm{e}^{j^{\prime}}$ is square-integrable since $j^{\prime} \leq j$. So $h \in \operatorname{dom} M_{+}(\kappa) \backslash\{0\}$, whence $\kappa$ is proper. -Next turn to $\Leftarrow$ for $p=\infty$. For $j^{\prime}$ from above $|\kappa| \mathrm{e}^{j^{\prime}} \leq|\kappa| \mathrm{e}^{j} \mathrm{e}^{-|x|^{1 / 2}}$ is square-integrable. Hence $\kappa$ is proper by the case $p=2$ just shown. -Now consider $\Leftarrow$ for $p=1$. Due to the assumption $|\kappa| \mathrm{e}^{j}\left(1+x^{2}\right)^{-1}$ is integrable, whence $j^{\prime}\left(1+x^{2}\right)^{-1}$ is integrable for $j^{\prime}:=\ln \left(1+|\kappa| \mathrm{e}^{j}\right)$. Therefore $\left(1+|\kappa| \mathrm{e}^{j}\right) \mathrm{e}^{-j^{\prime}}=1$ implying $|\kappa| \mathrm{e}^{j-j^{\prime}} \leq 1$, whence the result by $\Leftarrow$ for $p=\infty$. 
Lemma $2.7 \kappa$ is proper if and only if $\kappa^{2}$ is proper. More generally, let $r>0$ and let $\kappa_{1}, \kappa_{2}$ be two symbols satisfying $\left|\kappa_{1}\right|=\left|\kappa_{2}\right|^{r}$. Then $\kappa_{1}$ is proper if and only if $\kappa_{2}$ is proper. Finally, if $\kappa_{1}$ and $\kappa_{2}$ are proper symbols then so are $\kappa_{1} \kappa_{2}$ and $\kappa_{1}+\kappa_{2}$.

Proof Let $\kappa^{2}$ be proper. Then $1+|\kappa|^{2}$ is proper and $|\kappa| \leq 1+|\kappa|^{2}$, whence $\kappa$ is proper. -Now let $\kappa$ be proper. Then by $2.6(p=2), \kappa \mathrm{e}^{j} \in \bar{L}^{2}(\mathbb{R})$ for some real-valued $j$ with integrable $j /\left(1+x^{2}\right)$. Since $\kappa^{\prime}:=|\kappa|^{2} \mathrm{e}^{2 j}$ is integrable, $\kappa^{\prime}$ is proper by $2.6(\Leftarrow$ for $p=1)$. Then $2.6(p=2)$ yields $\kappa^{\prime} \mathrm{e}^{j^{\prime}} \in L^{2}(\mathbb{R})$ for some real-valued $j^{\prime}$ with integrable $j^{\prime} /\left(1+x^{2}\right)$. Hence $|\kappa|^{2} \mathrm{e}^{2 j+j^{\prime}} \in L^{2}(\mathbb{R})$, whence the claim by $2.6(p=2)$.

The general case is easily reduced to the claim that, for $r>1$ and $\kappa \geq 0, \kappa$ is proper if and only if $\kappa^{r}$ is proper. So let $\kappa$ be proper. Let $n \in \mathbb{N}$ satisfy $r \leq 2^{n}$. By the foregoing result $\kappa^{2^{n}}$ is proper. Then $1+\kappa^{2^{n}}$ is proper and $\kappa^{r} \leq 1+\kappa^{2^{n}}$. Hence $\kappa^{r}$ is proper. Conversely, if $\kappa^{r}$ is proper, then $1+\kappa^{r}$ is proper and $\kappa \leq 1+\kappa^{r}$, whence $\kappa$ is proper.

Now we complete the proof of 2.6. Consider first the case $p \in] 0, \infty\left[\right.$. Let $\kappa \mathrm{e}^{j} \in$ $L^{p}(\mathbb{R})$ for some $j$. Then $|\kappa|^{p / 2} \mathrm{e}^{\frac{p}{2} j} \in L^{2}(\mathbb{R})$. Hence $|\kappa|^{p / 2}$ is proper by $2.6(p=2)$. The foregoing result applies, whence $\kappa$ is proper. The converse follows quite similarly. -Now let $p=\infty$ and let $\kappa$ be proper. As just shown, $\kappa e^{j}$ is integrable for some realvalued $j$ with integrable $j\left(1+x^{2}\right)^{-1}$. Then $|\kappa| \mathrm{e}^{j^{\prime \prime}} \leq 1$ for $j^{\prime \prime}:=j-\ln \left(1+|\kappa| \mathrm{e}^{j}\right)$ as shown in the proof of $2.6(\Leftarrow$ for $p=1)$.

Finally, let $\kappa_{1}, \kappa_{2}$ be proper. Apply 2.6 for $p=\infty$. So $\left|\kappa_{i}\right| \leq \mathrm{e}^{j_{i}}, i=1,2$. Then $\left|\kappa_{1} \kappa_{2}\right| \leq \mathrm{e}^{j_{1}+j_{2}}$. Assume without restriction $j_{i} \geq 0, i=1,2$. Then $\left|\kappa_{1}+\kappa_{2}\right| \leq$ $2 \mathrm{e}^{j_{1}+j_{2}}$.

Theorem $2.8 \kappa$ is proper if and only if $\frac{\ln (1+|\kappa|)}{1+x^{2}}$ is integrable.

Proof Let $\kappa$ be proper. Then $1+|\kappa|$ is proper, and by $2.6(p=\infty)$ one has $1+|\kappa| \leq \mathrm{e}^{j}$ for some real-valued $j$ with integrable $\frac{j}{1+x^{2}}$. Then $\ln (1+|\kappa|) \leq j$, whence the claim. Conversely let $\frac{j}{1+x^{2}}$ be integrable for $j:=\ln (1+|\kappa|)$. Then $1+|\kappa|=\mathrm{e}^{j}$, whence $|\kappa| \leq \mathrm{e}^{j}$ and $\kappa$ is proper by $2.6(p=\infty)$.

Corollary $2.9 \kappa$ is not proper if $|\kappa(x)|$ increases not less than exponentially for $x \rightarrow \infty$ or $x \rightarrow-\infty$, i.e., if there are positive constants $a, \delta, \lambda$ such that $|\kappa(x)| \geq \delta \mathrm{e}^{\lambda|x|}$ for $x \geq a$ or $x \leq-a$.

Proof $\ln (1+|\kappa(x)|) \geq \ln |\kappa(x)| \geq \ln (\delta)+\lambda|x|$ for all $x \geq a$ or $x \leq-a$. Hence $\ln (1+|\kappa|) /\left(1+x^{2}\right)$ is not integrable. Apply 2.8 .

Now the alternative is shown that a WH is either trivial or densely defined.

Theorem 2.10 If $\kappa$ proper, then $\operatorname{dom} W_{\kappa}$ is dense. More precisely, if $h \in \operatorname{dom} M_{+}(\kappa) \backslash$ $\{0\}$, then there is $h^{\prime} \in H_{+}$with $|h|=\left|h^{\prime}\right|$ such that $H_{+}^{\infty} \frac{1}{x+\mathrm{i}} h^{\prime}$ is dense and contained in $\operatorname{dom} M_{+}(\kappa)$.

Proof Let $h \in \operatorname{dom} M_{+}(\kappa) \backslash\{0\}$. Then $\kappa h \in L^{2}(\mathbb{R})$, whence $\kappa \mathrm{e}^{j} \in L^{2}(\mathbb{R})$ for $j:=\ln |h|$. By (2.1), $j /\left(1+x^{2}\right)$ is integrable.

Recall 2.2. Put $c:=C^{-1}$. Check that $j \circ c \geq 0$ is integrable on the torus $\mathbb{T}$. Hence $U(w):=\exp \left(\frac{1}{2 \pi} \int_{0}^{2 \pi} \frac{\mathrm{e}^{\mathrm{i} t}+w}{\mathrm{e}^{\mathrm{i} t}-w} j \circ c\left(\mathrm{e}^{\mathrm{i} t}\right) \mathrm{d} t\right)$ is an outer function on the disc $\mathbb{D}$ (see e.g. 
[17, Chapter 3]). So $U$ converges non-tangentially a.e. to a function $u$ on $\mathbb{T}$ satisfying $|u|=\mathrm{e}^{j \circ c} \in L^{2}(\mathbb{T})$, whence $U \in H^{2}(\mathbb{D})$. Therefore, as known (see e.g. [21, Sect. 3]), $U H^{\infty}(\mathbb{D})$ is dense in $H^{2}(\mathbb{D})$. This implies that $u H^{\infty}(\mathbb{T})$ is dense in $H^{2}(\mathbb{T})$.

Put $h^{\prime}:=u \circ C$. Note $\left|h^{\prime}\right|=\mathrm{e}^{j}=|h|$. The above result is transferred to $H_{+}$ by $\Gamma$ in 2.2. Accordingly, $\frac{1}{x+\mathrm{i}} h^{\prime} \in H_{+}$and $\frac{1}{x+\mathrm{i}} h^{\prime} H_{+}^{\infty}$ is dense in $H_{+}$. The latter is contained in dom $M_{+}(\kappa)$ by 2.5 (a), since $\kappa \frac{1}{x+\mathrm{i}} h^{\prime}$ is square-integrable and hence $\frac{1}{x+\mathrm{i}} h^{\prime} \in \operatorname{dom} M_{+}(\kappa)$. Finally $h^{\prime} \in H_{+}$by $3.1(\mathrm{c})$.

Two results on the eigenvalues of WH follow. The proof of 2.11 uses an alternative argument with respect to $[22,2.8]$ for the case of bounded $\kappa$.

Theorem 2.11 Let $\kappa$ be not almost constant. If $\lambda \in \mathbb{C}$ is an eigenvalue of $W_{\kappa}$, then $\bar{\lambda}$ is not an eigenvalue of $W_{\bar{\kappa}}$. If $\kappa$ is almost real, then $W_{\kappa}$ has no eigenvalues and in particular $W_{\kappa}$ is injective.

Proof Keep 2.1 in mind. Since $W_{\kappa}-\lambda I=W\left(\kappa-\lambda 1_{\mathbb{R}}\right)$ assume without restriction $\lambda=0$. Suppose $W_{\kappa} u=0, W_{\bar{\kappa}} v=0$. Set $h_{+}:=\mathcal{F}^{-1} P_{+}^{*} u$. Then $h_{+} \in H_{+}$and $h_{-}:=\kappa h_{+} \in H_{-}$. Set $k_{-}:=\overline{\mathcal{F}^{-1} P_{+}^{*} v}$. Then $k_{-} \in H_{-}$and $\bar{\kappa} \bar{k}_{-} \in H_{-}$, whence $k_{+}:=\kappa k_{-} \in H_{+}$.

Note that $j:=h_{-} k_{-}=\kappa h_{+} k_{-}=h_{+} k_{+}$. Hence there is a holomorphic $\chi$ : $\mathbb{C} \backslash \mathbb{R} \rightarrow \mathbb{C}$ such that its partial maps satisfy $\chi_{y} \in L^{1}(\mathbb{R}), K:=\sup \left\{\left\|\chi_{y}\right\|_{1}\right.$ : $y \neq 0\}<\infty, \chi_{y} \rightarrow j$ pointwise a.e. and $\left\|\chi_{y}-j\right\|_{1} \rightarrow 0$ for $y \rightarrow 0$. By a standard argument (see also [23, Theorem II]) $\chi$ extends to an entire function still called $\chi$ with $\left.\chi\right|_{\mathbb{R}}=j$ a.e. Fix $z \in \mathbb{C},|z|>1$. We use the representation $\pi \chi(z)=$ $\int_{\mathbb{D}} \chi(z+w) \mathrm{d}^{2} w$. Then $\pi|\chi(z)| \leq \int_{-1}^{1} \int_{-\infty}^{\infty}|\chi(x+u+\mathrm{i}(y+v))| \mathrm{d} u \mathrm{~d} v \leq 2 K$ so that $\chi$ is constant equal to 0 , whence $h_{-} k_{-}=0, h_{+} k_{+}=0$. ( [22, 2.8] obtains this result using alternatively $\left[14\right.$, Theorem 76], by which $(2 \pi)^{1 / 2} \mathcal{F}_{L^{1}}\left(h_{ \pm} k_{ \pm}\right)=\mathcal{F} h_{ \pm} \star \mathcal{F} k_{ \pm}$. Accordingly, $\mathcal{F} h_{-} \star \mathcal{F} k_{-}=\mathcal{F} h_{+} \star \mathcal{F} k_{+}=0$, since $\mathcal{F} h_{-}, \mathcal{F} k_{-}$vanish on $[0, \infty[$ and $\mathcal{F} h_{+}, \mathcal{F} k_{+}$vanish on $\left.]-\infty, 0\right]$.) The cases $k_{-}=0$ or $h_{+}=0$ are trivial. Otherwise $h_{+} \neq 0$ and $\kappa h_{+}=h_{-}=0$, whence $\kappa=0$ a.e.

Now let $\kappa$ be real, let $\lambda \in \mathbb{C}$, and let $g \in \operatorname{dom} W_{\kappa}$ satisfy $W_{\kappa} g=\lambda g$. Then $\lambda\langle g, g\rangle=\left\langle g, W_{\kappa} g\right\rangle=\left\langle W_{\kappa} g, g\right\rangle=\bar{\lambda}\langle g, g\rangle$. Hence $g=0$, since otherwise $\lambda \in \mathbb{R}$ would contradict the foregoing result.

Proposition 2.12 Let $W_{\kappa}$ be not a multiple of I. If $\lambda \in \mathbb{C}$ is an eigenvalue of $W_{\kappa}$, then $\kappa \neq \lambda$ a.e.

Proof Put $E:=\kappa^{-1}(\{\lambda\}) . \mathbb{R} \backslash E$ is not a null set. Let $h \in \operatorname{dom} M_{+}(\kappa) \backslash\{0\}$ satisfy $M_{+}(\kappa) h=\lambda h$. Then $M_{+}\left(\kappa^{\prime}\right) h=0$ for $\kappa^{\prime}:=\kappa-\lambda$. Hence $h_{-}:=\kappa^{\prime} h \in H_{-}$. If $E$ were not a null set, then $h_{-}=0$ as vanishing on $E$, whence $h$ vanishes on $\mathbb{R} \backslash E$ implying the contradiction $h=0$.

The following result from $[12,3.2]$ obviously is stronger than 2.12: Let $\lambda$ be an eigenvalue of $W_{\kappa} \neq \lambda I$. Then $\frac{\ln |\kappa-\lambda|}{1+x^{2}}$ is integrable. The converse still does not hold as the case of real-valued symbols shows by 2.11 .

By 2.13 the symbol of a WH is uniquely determined. 
Lemma 2.13 Let $W_{\kappa_{1}}$ and $W_{\kappa_{2}}$ coincide on a dense set of $L^{2}\left(\mathbb{R}_{+}\right)$. Then $\kappa_{1}=\kappa_{2}$ a.e. In particular, if $\kappa$ is proper and $W_{\kappa} \subset 0$, then $\kappa=0$ a.e. and $W_{\kappa}=0$.

Proof Put $\beta:=\kappa_{1}-\kappa_{2}$. Then $\left.W_{\beta}\right|_{D}=0$ for some dense $D \subset L^{2}\left(\mathbb{R}_{+}\right)$. Let $k \in$ $\operatorname{dom} W_{\beta}^{*}$. Then for all $g \in D$ one has $0=\left\langle k, W_{\beta} g\right\rangle=\left\langle W_{\beta}^{*} k, g\right\rangle$, whence $W_{\beta}^{*} k=0$. Since $W_{\bar{\beta}} \subset W_{\beta}^{*}$, it follows $\left.W_{\bar{\beta}}\right|_{D}=0$. So $\beta$ is almost constant by 2.11 , whence $\beta=0$ a.e.

We turn to symmetric WH.

Corollary 2.14 Let $W_{\kappa}$ be densely defined. Then $W_{\kappa}$ is symmetric if and only if $\kappa$ is almost real.

Proof Let $W_{\kappa} \subset W_{\kappa}^{*}$. Since generally $W_{\bar{\kappa}} \subset W_{\kappa}^{*}$ and dom $W_{\kappa}=\operatorname{dom} W_{\bar{\kappa}}$, it follows $W_{\kappa}=W_{\bar{\kappa}}$, whence $\kappa=\bar{\kappa}$ a.e. by 2.13 . The converse is obvious.

Hence due to 2.11 densely defined symmetric WH have no eigenvalues. - Now the numerical range of densely defined semibounded WH is determined.

Theorem 2.15 Let $W_{\kappa}$ be densely defined symmetric. Then $W_{\kappa}$ is bounded below if and only if $\kappa$ is real essentially bounded below, and the maximal lower bound of $W_{\kappa}$ equals the maximal essential lower bound of $\kappa$. If $W_{\kappa}$ is bounded below and not bounded, then the numerical range $\left\{\left\langle g, W_{\kappa} g\right\rangle: g \in \operatorname{dom} W_{\kappa},\|g\|=1\right\}$ equals $] \alpha, \infty[$ with $\alpha$ the maximal lower bound.

Proof By 2.14 let $\kappa$ be real. First suppose that $\kappa$ is bounded below with maximal lower bound $a$. Put $\kappa^{\prime}:=\kappa-a \geq 0$. Then for all $h \in \operatorname{dom} M_{+}\left(\kappa^{\prime}\right)=\operatorname{dom} M_{+}(\kappa)$ one has $\left\langle h, M_{+}\left(\kappa^{\prime}\right) h\right\rangle=\int \kappa^{\prime}|h|^{2} \mathrm{~d} x \geq 0$, whence $\left\langle h, M_{+}(\kappa) h\right\rangle \geq a\|h\|^{2}$. So $a$ is a lower bound for $M_{+}(\kappa)$.

Now let $M_{+}(\kappa)$ be bounded below with maximal lower bound $\alpha$. Put $\kappa^{\prime}:=\kappa-$ $\alpha$. Then $\left\langle h, M_{+}\left(\kappa^{\prime}\right) h\right\rangle \geq 0 \forall h \in \operatorname{dom} M_{+}\left(\kappa^{\prime}\right)$. We show that $B:=\{x \in \mathbb{R}:$ $\left.\kappa^{\prime}(x)<0\right\}$ is a null set. Assume the contrary. Let $h_{0} \in \operatorname{dom} M_{+}\left(\kappa^{\prime}\right) \backslash\{0\}$. Then $c:=\int_{B} \kappa^{\prime}\left|h_{0}\right|^{2} \mathrm{~d} x<0$, since $h_{0}$ does not vanish on a set of positive measure. Put $A:=\mathbb{R} \backslash B$ and let $\lambda \in] 0,1\left[\right.$. Then $f:=\lambda 1_{A}\left|h_{0}\right|+1_{B}\left|h_{0}\right| \leq\left|h_{0}\right|$. Hence $f \in L^{2}(\mathbb{R}) \backslash\{0\},|\ln f| \leq|\ln | h_{0}||$, and $\ln f /\left(1+x^{2}\right)$ is integrable by (2.1). Therefore by (2.1) there is $h \in H_{+}$with $|h|=|f|$. Note that $h \in \operatorname{dom} M_{+}\left(\kappa^{\prime}\right)$, since $\left|\kappa^{\prime} h\right|^{2} \leq$ $\left|\kappa^{\prime} h_{0}\right|^{2}$ is integrable. Hence the contradiction $0 \leq\left\langle h, M_{+}\left(\kappa^{\prime}\right) h\right\rangle=\int \kappa^{\prime}|h|^{2} \mathrm{~d} x=$ $\lambda^{2} \int_{A} \kappa^{\prime}\left|h_{0}\right|^{2} \mathrm{~d} x+c<0$ follows for $\lambda$ sufficiently small. Therefore $B$ is a null set, whence $\kappa^{\prime} \geq 0$ a.e. and $\alpha$ is an essential lower bound of $\kappa$. - This proves $a=\alpha$.

Since $M_{+}(\kappa)$ is not bounded above by assumption, the numerical range $R$ of $M_{+}(\kappa)$ is not bounded above. As $R$ is convex one infers ] $\alpha, \infty[\subset R \subset[\alpha, \infty$ [. It remains to show $\alpha \notin R$ or, equivalently, that $\left\langle h, M_{+}\left(\kappa^{\prime}\right) h\right\rangle=0$ implies $h=0$, where $\kappa^{\prime} \geq 0$ is not almost zero. Indeed, $0=\left\langle h, M_{+}\left(\kappa^{\prime}\right) h\right\rangle=\int \kappa^{\prime}|h|^{2} \mathrm{~d} x \Rightarrow h$ vanishes on a non-null set $\Rightarrow h=0$.

For bounded WH, 2.15 implies the following well-known result.

Corollary 2.16 Let $W_{\kappa}$ be bounded symmetric and not a multiple of I. Then the numerical range equals] $a, b$ [ with $a$ and $b$ the minimum and maximum, respectively, of the essential range of $\kappa$. 
Concluding this section we deal with the unilateral translation invariance of $\mathrm{WH}$. We are inspired by [6, Sect.2.3)] which treats the bounded case 2.18. Observe the easily verifiable relation

$$
T_{b}^{*} P_{+}^{*} W_{\kappa} P_{+} T_{b}=M\left(1_{]-b, \infty[}\right) \mathcal{F} M(\kappa) \mathcal{F}^{-1} M\left(1_{]-b, \infty[}\right)
$$

where $T_{b}, b \in \mathbb{R}$ denotes the unitary one-parameter group of translations $T_{b} f(x):=$ $f(x-b)$ on $L^{2}(\mathbb{R})$. It shows again the invariance $2.5(\mathrm{c})$

$$
W_{\kappa}=S_{b}^{*} W_{\kappa} S_{b}
$$

under the unilateral translations $S_{b}, b \geq 0$, since $S_{b}=P_{+} T_{b} P_{+}^{*}$. Recall $S_{b}=$ $W\left(\mathrm{e}^{\mathrm{i} b(\cdot)}\right)$. Moreover it implies that $T_{b}^{*} P_{+}^{*} W_{\kappa} P_{+} T_{b} f$ converges as $b \rightarrow \infty$ if $f \in T_{a}^{*} P_{+}^{*}\left(\operatorname{dom} W_{\kappa}\right)$ for some $a \geq 0$, yielding the limit $\mathcal{F} M(\kappa) \mathcal{F}^{-1} f$. In particular $\left\{\left\|W_{\kappa} S_{b} g\right\|: b \geq 0\right\}$ is bounded for every $g \in \operatorname{dom} W_{\kappa}$. If $W_{\kappa}$ is densely defined then also $\left\{\left\|W_{\kappa}^{*} S_{b} g\right\|: b \geq 0\right\}$ is bounded as $\left.W_{\kappa}^{*}\right|_{\operatorname{dom} W_{\kappa}}=W_{\bar{\kappa}}$.

Theorem 2.17 Let A be a densely defined operator in $L^{2}\left(\mathbb{R}_{+}\right)$satisfying

$$
A \subset S_{b}^{*} A S_{b} \quad \forall b \geq 0
$$

If $\operatorname{dom} A \subset \operatorname{dom} A^{*}$ and $\left\{\left\|A S_{b} g\right\|: b \geq 0\right\}$ and $\left\{\left\|A^{*} S_{b} g\right\|: b \geq 0\right\}$ are bounded for every $g \in \operatorname{dom} A$ then there is a WH $W_{\kappa}$ extending $A$.

Proof (i) For $a \geq 0$ put $D_{a}:=T_{a}^{*} P_{+}^{*}(\operatorname{dom} A)$ and $D:=\bigcup_{a \geq 0} D_{a}$. Then $D$ is a dense translation invariant subspace of $L^{2}(\mathbb{R})$.

Indeed, let $f \in L^{2}(\mathbb{R})$ and $\varepsilon>0$. Since $f_{a}:=1_{]-a, \infty[} f \rightarrow f$ for $a \rightarrow \infty$ in the mean, one has $\left\|f-f_{a}\right\| \leq \varepsilon / 2$ for some $a \geq 0$. As $T_{a} f_{a}=P_{+}^{*} P_{+} T_{a} f$, there is $g \in \operatorname{dom} A$ with $\left\|P_{+} T_{a} f_{a}-g\right\| \leq \varepsilon / 2$. Hence $\left\|f_{a}-T_{a}^{*} P_{+}^{*} g\right\| \leq \varepsilon / 2$. It follows that $D$ is dense.

Note that $S_{b}(\operatorname{dom} A) \subset \operatorname{dom} A$ for $b \geq 0$ due to $A \subset S_{b}^{*} A S_{b}$. Then $D_{a} \subset D_{b}$ for $a \leq b$. Indeed, put $c:=b-a \geq 0$. Then check $T_{a}^{*} P_{+}^{*}=T_{b}^{*} P_{+}^{*} S_{c}$, whence $D_{a}=T_{b}^{*} P_{+}^{*} S_{c}(\operatorname{dom} A) \subset T_{b}^{*} P_{+}^{*}(\operatorname{dom} A)=D_{b}$. It follows that $D$ is a subspace of $L^{2}(\mathbb{R})$.

For the translation invariance of $D$ it suffices to show $T_{c}^{*} P_{+}^{*} g \in D$ for $c \in \mathbb{R}$, $g \in \operatorname{dom} A$. If $c \geq 0$ this is obvious. Let $c<0$. Then $T_{c}^{*} P_{+}^{*} g=T_{-c} P_{+}^{*} g=$ $P_{+}^{*} P_{+} T_{-c} P_{+}^{*} g=P_{+}^{*} S_{-c} g \in D$ as $S_{-c} g \in \operatorname{dom} A$.

(ii) Let $f \in D$. Then $f=T_{a}^{*} P_{+}^{*} g$ for some $a \geq 0, g \in \operatorname{dom} A$, whence $f_{b}:=$ $T_{b}^{*} P_{+}^{*} A P_{+} T_{b} f=T_{b}^{*} P_{+}^{*} A S_{b-a} g$ is well-defined for all $b \geq a$. We are going to show that $\lim _{b \rightarrow \infty} f_{b}$ exists.

Let $c \geq b \geq a$ : $\left\|f_{c}-f_{b}\right\|^{2}=\left\|f_{c}\right\|^{2}+\left\|f_{b}\right\|^{2}-\left\langle f_{c}, f_{b}\right\rangle-$ $\left\langle f_{b}, f_{c}\right\rangle$, with $\left\|f_{c}\right\|^{2}=\left\|A P_{+} T_{c} f\right\|^{2},\left\|f_{b}\right\|^{2}=\left\|A P_{+} T_{b} f\right\|^{2},\left\langle f_{c}, f_{b}\right\rangle=$ $\left\langle S_{c-b}^{*} A P_{+} T_{c-b} T_{b} f, A P_{+} T_{b} f\right\rangle=\left\langle S_{c-b}^{*} A P_{+} T_{c-b}\left(P_{+}^{*} P_{+} T_{b} f\right), A P_{+} T_{b} f\right\rangle=$ $\left\langle S_{c-b}^{*} A S_{c-b} P_{+} T_{b} f, A P_{+} T_{b} f\right\rangle$, which equals $\left\|A P_{+} T_{b} f\right\|^{2}$ by (2.6) since $P_{+} T_{b} f \in \operatorname{dom} A$. So $\left\|f_{c}-f_{b}\right\|^{2}=\left\|A P_{+} T_{c} f\right\|^{2}-\left\|A P_{+} T_{b} f\right\|^{2}$. This implies 
that $b \mapsto\left\|A P_{+} T_{b} f\right\|^{2}$ is increasing. Being bounded by the assumption it follows $\left\|f_{c}-f_{b}\right\|^{2} \rightarrow 0$ for $b, c \rightarrow \infty$ so that $\lim _{b \rightarrow \infty} f_{b}$ exists.

(iii) Thus $C_{1} f:=\lim _{b \rightarrow \infty} f_{b}$ defines an operator $C_{1}$ on $D$. Since one has $T_{c}^{*} C_{1} T_{c} f=\lim _{b \rightarrow \infty} T_{c}^{*} T_{b}^{*} P_{+}^{*} A P_{+} T_{b} T_{c} f=\lim _{b \rightarrow \infty} T_{c+b}^{*} P_{+}^{*} A P_{+} T_{c+b} f=$ $\lim _{b^{\prime} \rightarrow \infty} T_{b^{\prime}}^{*} P_{+}^{*} A P_{+} T_{b^{\prime}} f=C_{1} f$, it is translation invariant.

(iv) Also $C_{2} f:=\lim _{b \rightarrow \infty} T_{b}^{*} P_{+}^{*} A^{*} P_{+} T_{b} f$ exists for $f \in D$ thus defining an operator $C_{2}$ on $D$. This result follows replacing $A$ in (ii) by $A^{\#}:=\left.A^{*}\right|_{\operatorname{dom} A}$. It remains to verify $A^{\#} \subset S_{b}^{*} A^{\#} S_{b}, b \geq 0$. Indeed, for $g, g^{\prime} \in \operatorname{dom} A$ one has $\left\langle g, S_{b}^{*} A^{\#} S_{b} g^{\prime}\right\rangle=\left\langle S_{b} g, A^{*} S_{b} g^{\prime}\right\rangle=\left\langle A S_{b} g, S_{b} g^{\prime}\right\rangle$ since $S_{b} g \in \operatorname{dom} A \subset$ $\operatorname{dom} \bar{A}=\operatorname{dom} A^{* *}$. So $\left\langle g, S_{b}^{*} A^{\#} S_{b} g^{\prime}\right\rangle=\left\langle S_{b}^{*} A S_{b} g, g^{\prime}\right\rangle=\left\langle A g, g^{\prime}\right\rangle=\left\langle g, A^{\#} g^{\prime}\right\rangle$ by (2.6) and $g, g^{\prime} \in \operatorname{dom} A$. This implies $S_{b}^{*} A^{\#} S_{b} g^{\prime}=A^{\#} g^{\prime}$, whence the claim.

(v) Obviously $C_{2} \subset C_{1}^{*}$. Hence $C_{1}^{*}$ is densely defined and the closure $C:=\bar{C}_{1}$ exists. Clearly translation invariance $C=T_{b}^{*} C T_{b}$ holds. Equivalently $\mathcal{F}^{-1} C \mathcal{F}$ commutes with $M\left(\mathrm{e}^{\mathrm{i} b(\cdot)}\right)=\mathcal{F}^{-1} T_{b} \mathcal{F}$ for all $b \in \mathbb{R}$. Thus $\mathcal{F}^{-1} C \mathcal{F}=M(\kappa)$ for some measurable function $\kappa$. Hence $P_{+} C P_{+}^{*}=W_{\kappa}$. Finally, for $g \in \operatorname{dom} A$ one has $P_{+}^{*} g \in D, W_{\kappa} g=P_{+} C_{1} P_{+}^{*} g=\lim _{b \rightarrow \infty} P_{+} T_{b}^{*} P_{+}^{*} A P_{+} T_{b} P_{+}^{*} g=$ $\lim _{b \rightarrow \infty} S_{b}^{*} A S_{b} g=\lim _{b \rightarrow \infty} A g=A g$.

Corollary 2.18 Let A be a bounded operator on $L^{2}\left(\mathbb{R}_{+}\right)$. Then A is a WH if and only if

$$
A=S_{b}^{*} A S_{b} \quad \forall b \geq 0
$$

Proof It remains to observe that $A \subset W_{\kappa}$ by 2.17 implies $A=W_{\kappa}$.

For 2.18 see also [6, (2.10)], where the existence of $\lim _{b \rightarrow \infty} T_{b}^{*} P_{+}^{*} A P_{+} T_{b} f$ (see (ii) of the proof of 2.17) is not proven.

\section{Rational Symbols}

WH for rational symbols $\kappa=\left.\frac{P}{Q}\right|_{\mathbb{R}}$ with polynomials $P \neq 0, Q \neq 0$ permit some more general analysis. By 2.4 they are densely defined. In 3.2 we show that they are closed and we determine their domains, ranges, and kernels and deficiency spaces, which are finite dimensional, and their spectral and Fredholm points. In particular, in the symmetric case, i.e., for a real rational symbol the deficiency spaces and indices are explicitly available 3.4 .

Recall that a densely defined closed operator between Banach spaces with finite dimensional kernel and cokernel is called a Fredholm operator if its range is closed (cf. [25]).

Mostly we will omit $\left.\right|_{\mathbb{R}}$ indicating the restriction on $\mathbb{R}$. A polynomial with a negative degree is the null function. For convenience we will deal with $M_{+}(\kappa)(2.2)$ in place of $W_{\kappa}$. We are indebted to the reviewer for a significantly simplified proof of (b) and (c) of the following lemma 3.1 . 
Lemma 3.1 Let $P \neq 0$ and $Q \neq 0$ be polynomials.

(a) Let $P$ and $Q$ have no common zeros. Then $\frac{P}{Q} \in H_{+}\left(\in H_{-}\right)$if and only if $\operatorname{deg} P<\operatorname{deg} Q$ and all zeros of $Q$ are in the lower (upper) half-plane.

(b) Let $h \in H_{+} \backslash\{0\}$ such that $\frac{P}{Q} h \in H_{-}$. Then there is a polynomial $R$ with $\operatorname{deg} R<$ $\min \{\operatorname{deg} P, \operatorname{deg} Q\}$ such that all zeros of $P$ in the closed upper half-plane as well as all zeros of $Q$ in the closed lower half-plane are zeros of $R$ with at least the same multiplicities and such that $h=\frac{R}{P}$. Conversely, it is obvious that $h_{+}:=\frac{R}{P}$ and $h_{-}:=\frac{R}{Q}$ satisfy $h_{ \pm} \in H_{ \pm}$and $\frac{P}{Q} h_{+}=h_{-}$.

(c) Let $Q$ have no zeros in the upper half-plane. If $h \in H_{+}$and $\frac{P}{Q} h \in L^{2}(\mathbb{R})$, then $\frac{P}{Q} h \in H_{+}$.

(d) Let $P$ and $Q$ have no common zeros. Suppose that $h \in H_{+}$and $\frac{P}{Q} h \in H_{+}$. Then $h / Q \in H_{+}$.

Proof (a) The result is known and we omit the proof.

(b) Put $n:=\max \{\operatorname{deg} P, \operatorname{deg} Q\}, k:=\frac{P}{Q} h, \alpha:=(x+\mathrm{i})^{-n} P, \beta:=(x-\mathrm{i})^{-n} Q$. Then $\alpha h \in H_{+}$and $\beta k \in H_{-}$, since $\alpha \in H_{+}^{\infty}, \beta \in H_{-}^{\infty}$, and

$$
\left(\frac{x+\mathrm{i}}{x-\mathrm{i}}\right)^{n} \alpha h=\beta k
$$

Hence $\alpha h \in \operatorname{ker} M_{+}\left(\left(\frac{x+\mathrm{i}}{x-\mathrm{i}}\right)^{n}\right)$, whence $\alpha h=(x+\mathrm{i})^{-n} R$ for some polynomial $R$ with $\operatorname{deg} R<n$ by [22, Theorem 3.1]. Therefore $h=R / P$ and $k=R / Q$. Now, $h \in H_{+}$implies by (a) that $\operatorname{deg} R<\operatorname{deg} P$ and all zeros of $P$ in the closed upper half-plane are zeros of $R$ with at least the same multiplicities. The analogous result regarding $Q$ follows from $k \in H_{-}$.

(c) For $g:=\frac{P}{Q} h \in L^{2}(\mathbb{R})$ write $g=g_{+}+g_{-}$with $g_{+} \in H_{+}, g_{-} \in H_{-}$. Then, using $n, \alpha, \beta$ from the proof of (b) and $\gamma:=(x+\mathrm{i})^{-n} Q$, it follows

$$
\left(\frac{x+\mathrm{i}}{x-\mathrm{i}}\right)^{n}\left(\alpha h-\gamma g_{+}\right)=\beta g_{-}
$$

whence analogously $P h-Q g_{+}=Q g_{-}=R$ for some polynomial $R$. Then $g_{-}=R / Q$ implies $R=0$ by (a), since $Q$ has no zeros in the upper half-plane by assumption.

(d) Assume first that $Q$ has no real zeros. Let $\phi$ and $\psi$ be the holomorphic functions on the upper half-plane related to $h$ and $\frac{P}{Q} h$, respectively. Then $\frac{P}{Q} \phi$ is meromorphic on the upper half-plane and converges non-tangentially to $\frac{P}{Q} h$ a.e. Since $\psi$ does the same, according to [16, IV 2.5], $\psi=\frac{P}{Q} \phi$ holds. Hence $\phi / Q$ is holomorphic on the upper half-plane with $(\phi / Q)_{y} \rightarrow h / Q$ for $0<y \rightarrow 0$ a.e. Let $0<\delta<c$ such that $C:=[-c, c] \times \mathrm{i}[\delta, c]$ is a neighborhood of the zeros of $Q$ in the upper half-plane. Then $|1 / Q|$ is bounded by some constant $L$ on $\{z: \operatorname{Im} z \geq 0\} \backslash C$, and $|\phi / Q|$ is bounded on $C$ by some $M$. Recall that $\left\|\phi_{y}\right\|_{2}^{2}$ is bounded for $y>0$ by some $K$. Then $\int|(\phi / Q)(x+\mathrm{i} y)|^{2} \mathrm{~d} x \leq L^{2} \int|\phi(x+\mathrm{i} y)|^{2} \mathrm{~d} x+\int_{-c}^{c} M^{2} \mathrm{~d} x \leq L^{2} K+2 c M^{2}$ 
for all $y>0$. Finally, for $0<y<\delta, \int|(\phi / Q)(x+\mathrm{i} y)-(h / Q)(x)|^{2} \mathrm{~d} x \leq$ $\left.L^{2} \int \mid \psi(x+\mathrm{i} y)-h\right)\left.(x)\right|^{2} \mathrm{~d} x \rightarrow 0$ for $y \rightarrow 0$, whence $h / Q \in H_{+}$.

Now we turn to the general case. Note $h / Q \in L^{2}(\mathbb{R})$, cf. (v). Write $Q=q Q_{0}$, where $q$ has only real zeros and $Q_{0}$ has no real zeros. Let $q_{\epsilon}(z):=q(z+\mathrm{i} \epsilon)$ for $\epsilon>0$ and put $Q_{\epsilon}:=q_{\epsilon} Q_{0}$. Note $\left|q(z) / q_{\epsilon}(z)\right|<1$ on the upper half-plane. Therefore $\frac{P}{Q_{\epsilon}} h=\frac{q}{q_{\epsilon}} \frac{P}{Q} h \in H_{+}$, where $Q_{\epsilon}$ has no real zeros. Moreover, for $\epsilon>0$ small enough, $P$ and $Q_{\epsilon}$ have no common zeros. Hence the foregoing result applies so that $h / Q_{\epsilon} \in H_{+}$. Now $h / Q_{\epsilon}=\frac{q}{q_{\epsilon}} h / Q \rightarrow h / Q$ for $\epsilon \rightarrow 0$ in the mean implying $h / Q \in H_{+}$.

Let $\kappa=\frac{P}{Q}$ be a rational function, where the polynomials $P$ and $Q$ have no common zeros. Then let $q$ be the polynomial, the zeros of which are the real zeros of $Q$ with the same multiplicities. Put $\varsigma:=\max \{\operatorname{deg} q, \operatorname{deg} P-\operatorname{deg} Q+\operatorname{deg} q\}$. Moreover put $P=P_{<} P_{>}$, where the zeros of $P_{<}\left(P_{>}\right)$and $P_{>}$are exactly the zeros of $P$ in the lower(upper) half-plane and in the closed upper half-plane, respectively. $\tilde{P}$ denotes the polynomial whose coefficients are the complex conjugates of $P$. Analogous notations concern $Q$.

Theorem 3.2 Let $\kappa=\frac{P}{Q}$ be a rational function, where the polynomials $P$ and $Q$ have no common zeros. Then $M_{+}(\kappa)$ is densely defined and closed and

(a) $\operatorname{dom} M_{+}(\kappa)=\frac{q}{(x+\mathrm{i})^{5}} H_{+}$and $\operatorname{ran} M_{+}(\kappa)=P_{H_{+}}\left(\frac{P}{Q_{>} Q_{<}(x+\mathrm{i})^{5}} H_{+}\right)$

(b) $\operatorname{ker} M_{+}(\kappa)=\left\{\left(Q_{\leq} / P_{<}\right) r: \quad r\right.$ polynomial with $\operatorname{deg} r<\min \left\{\operatorname{deg} P_{<}-\right.$ $\left.\left.\operatorname{deg} Q_{\leq}, \operatorname{deg} Q_{>}-\operatorname{deg} P_{\geq}\right\}\right\}$

(c) $\left(\operatorname{ran} M_{+}(\kappa)\right)^{\perp}=\left\{\left(\tilde{Q}_{<} / \widetilde{P}_{<}\right) r: r\right.$ polynomial with $\left.\operatorname{deg} r<\operatorname{deg} P_{>}-\operatorname{deg} Q_{>}\right\}$

(d) the following statements are equivalent:

(1) $M_{+}(\kappa)$ is a Fredholm Operator

(2) $\operatorname{ran} M_{+}(\kappa)$ closed

(3) $\operatorname{deg} Q \leq \operatorname{deg} P$ and $P$ without real zeros

(4) $0 \notin \overline{\kappa(\mathbb{R})}$

Proof For the closeness of $M_{+}(\kappa)$ write $\frac{P}{Q}$ in the form $\frac{P}{Q}=\frac{P_{0}}{Q_{0}}+\frac{p}{q}$ with polynomials $P_{0}, Q_{0}, p, q$ such that $Q=Q_{0} q, Q_{0}$ has no real zeros, $q$ has only real zeros, $\operatorname{deg} P_{0}<$ $\operatorname{deg} Q_{0}$, and $p$ and $q$ have no common zeros and satisfy $\varsigma=\max \{\operatorname{deg} p, \operatorname{deg} q\}$.

Since $\kappa_{0}:=\left.\frac{P_{0}}{Q_{0}}\right|_{\mathbb{R}}$ is bounded, $M_{+}\left(\kappa_{0}\right)$ is bounded. It follows $M_{+}(\kappa)=M_{+}\left(\kappa_{0}\right)+$ $M_{+}\left(\frac{p}{q}\right)$ and it remains to show that $M_{+}\left(\frac{p}{q}\right)$ is closed. Let $h_{n} \in \operatorname{dom} M_{+}\left(\frac{p}{q}\right)$ such that $\left(h_{n}\right)$ converges to some $h \in H_{+}$and $\left(M_{+}\left(\frac{p}{q}\right) h_{n}\right)$ converges to some $k \in H_{+}$. By 3.1(c), $\frac{p}{q} h_{n} \in H_{+}$. Hence one has $h_{n} \rightarrow h$ and $\frac{p}{q} h_{n} \rightarrow k$ in $L^{2}(\mathbb{R})$. Since $M\left(\frac{p}{q}\right)$ is closed, $h \in \operatorname{dom} M_{+}(\kappa)$ and $k=M_{+}\left(\frac{p}{q}\right) h$ follows.

(a) dom $M_{+}(\kappa)$ is dense by 2.4. Arguing as above it remains to show dom $M_{+}\left(\frac{p}{q}\right)=$ $\frac{q}{(x+\mathrm{i})^{5}} H_{+}$. Let $h \in H_{+}$. Then, by 3.1(c), $\frac{q}{(x+\mathrm{i})^{5}} h \in H_{+}$and $\frac{p}{q} \frac{q}{(x+\mathrm{i})^{5}} h \in H_{+}$implying $\frac{q}{(x+\mathrm{i})^{5}} H_{+} \subset \operatorname{dom} M_{+}\left(\frac{p}{q}\right)$. For the converse inclusion argue $g \in \operatorname{dom} M_{+}\left(\frac{p}{q}\right)$ 
$\Rightarrow g \in H_{+}, \frac{p}{q} g \in L^{2} \Rightarrow h:=\frac{p}{q} g \in H_{+}$by 3.1(c). Hence $g=\frac{q}{p} h$, whence $\frac{1}{p} h \in$ $H_{+}$by $3.1(\mathrm{~d})$. Since $\varsigma=\max \{\operatorname{deg} p, \operatorname{deg} q\}$ one infers $k:=\frac{(x+\mathrm{i})^{\varsigma}}{p} h \in L^{2}$, whence $k \in H_{+}$applying 3.1(c) to $\frac{1}{p} h \in H_{+}$. This shows $g=\frac{q}{(x+\mathrm{i})^{\varsigma}} k \in \frac{q}{(x+\mathrm{i})^{5}} H_{+} \cdot-$ Now the claim about ran $M_{+}(\kappa)$ is obvious.

(b) Check: $h_{0} \in \operatorname{ker} M_{+}(\kappa) \Leftrightarrow h_{0} \in \operatorname{dom} M_{+}(\kappa), M_{+}(\kappa) h_{0}=0 \Leftrightarrow h_{0} \in H_{+}$, $\kappa h_{0} \in L^{2}(\mathbb{R})$, and $\kappa h_{0} \in H_{-}$. According to 3.1(b) this means $h_{0}=R / P$, where $R$ is a polynomial with $\operatorname{deg} R<\min \{\operatorname{deg} P, \operatorname{deg} Q\}$ and $R=P_{\geq} Q_{\leq} r$, whence the claim.

(c) Using (a) one has $h_{0} \in\left(\operatorname{ran} M_{+}(\kappa)\right)^{\perp} \Leftrightarrow 0=\left\langle h_{0}, \frac{P}{Q_{>} Q_{<}(x+\mathrm{i}) \varsigma} h\right\rangle=$ $\left\langle\frac{\tilde{P}}{\left(Q_{>}\right)^{\sim}\left(Q_{<}\right)^{\sim}(x-\mathrm{i})^{\varsigma}} h_{0}, h\right\rangle \forall h \in H_{+} \Leftrightarrow \frac{\tilde{P}}{\left(Q_{>}\right)^{\sim}\left(Q_{<}\right)^{\sim}(x-\mathrm{i})^{\zeta}} h_{0} \in H_{-}$. By 3.1(b) this means $h_{0}=R / \tilde{P}$, where $R$ is a polynomial such that $\operatorname{deg} R<\min \{\operatorname{deg} \tilde{P}, \operatorname{deg} \tilde{Q}-$ $\operatorname{deg} q+\varsigma\}=\operatorname{deg} P$ and $R=\tilde{P}_{\geq}\left(Q_{>}\right)^{\sim} r=\tilde{P}_{\geq} \tilde{Q}_{<} r$, whence the claim.

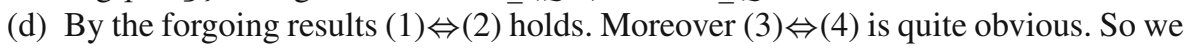
turn to $(2) \Leftrightarrow(3)$.

Show first (3) $\Rightarrow(2)$. Put $R:=\frac{P}{\left(Q_{>}\right)^{\sim} Q_{<}(x+\mathrm{i}) 5}$. Since by the assumptions nominator and denominator of $R$ have equal degree and have no real zeros, $R$ and $\frac{1}{R}$ are bounded on $\mathbb{R}$. Hence $M(R)$ is a homeomorphism on $L^{2}(\mathbb{R})$, whence $R H_{+}$is closed. Since $\left(Q_{>}\right)^{\sim}=\tilde{Q}_{<}$it follows by 3.1(c) that $R H_{+} \subset H_{+}$. Hence $R H_{+}=\operatorname{ran} M_{+}\left(\kappa^{\prime}\right)$ for $\kappa^{\prime}:=\frac{P}{Q^{\prime}}$ with $Q^{\prime}:=\tilde{Q}_{<} Q_{<q}$, whence $\operatorname{dim}\left(R H_{+}\right)^{\perp}<\infty$ by (c). So it suffices to show that $R H_{+} \subset \operatorname{ran} M_{+}(\kappa)$. By 3.1(c), $\frac{Q_{>}}{\bar{Q}_{<}} H_{+} \subset H_{+}$. So by (a), $\operatorname{ran} M_{+}(\kappa) \supset$ $P_{H_{+}}\left(\frac{P}{Q_{>} Q_{<}(x+\mathrm{i})^{5}} \frac{Q_{>}}{\left(Q_{>}\right)^{\sim}} H_{+}\right)=P_{H_{+}}\left(R H_{+}\right)=R H_{+}$.

Now turn to (2) $\Rightarrow(3)$. Consider first the case $\operatorname{deg} P<\operatorname{deg} Q$. Put $R:=Q_{>} Q_{<}(x+$

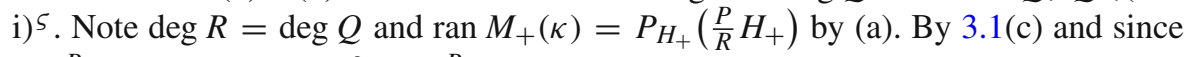
$M\left(\frac{R_{>}}{\widetilde{R}_{<}}\right)$is unitary on $L^{2}(\mathbb{R}), \frac{R_{>}}{\widetilde{R}_{<}} H_{+}$is a closed subspace of $H_{+}$. By (c) one has $\left(\frac{R_{>}}{R_{<}} H_{+}\right)^{\perp}=\left\{\frac{r}{R_{<}}: \operatorname{deg} r<\operatorname{deg} R_{>}\right\}$and hence

$$
\frac{P}{R} H_{+}=\left\{\frac{P r}{R \widetilde{R}_{<}}: \operatorname{deg} r<\operatorname{deg} R_{>}\right\}+\frac{P}{R_{<} \widetilde{R}_{<}} H_{+}
$$

Applying (c) for $\kappa=\frac{P}{R_{<} \widetilde{R}_{<}}$and $\kappa=\frac{P_{>}}{\widetilde{P}_{<}}$check $\left(\frac{P}{R_{<} \widetilde{R}_{<}} H_{+}\right)^{\perp}=\left(\frac{P_{>}}{\widetilde{P}_{<}} H_{+}\right)^{\perp}=\left\{\frac{r}{\widetilde{P}_{<}}\right.$: $\left.\operatorname{deg} r<\operatorname{deg} P_{>}\right\}$. Hence $\frac{P}{R_{<} \widetilde{R}_{<}} H_{+}$is dense in $\frac{P_{>}}{\widetilde{P}_{<}} H_{+}$, which is closed in $H_{+}$. Note that $P_{H_{+}}\left\{\frac{P r}{R \widetilde{R}_{<}}: \operatorname{deg} r<\operatorname{deg} R_{>}\right\} \subset V:=\left\{\frac{B}{R_{<} \widetilde{R}_{<}}: \operatorname{deg} B<\operatorname{deg} R\right\}$.

Now assume that $P_{H_{+}}\left(\frac{P}{R} H_{+}\right)$is closed. Then the above considerations imply that $\frac{P_{>}}{\widetilde{P}_{<}} H_{+} \subset V+\frac{P}{R_{<} \widetilde{R}_{<}} H_{+}$. Thus given $h_{0} \in H_{+}$there is $h \in H_{+}$and some polynomial $B$ with $\operatorname{deg} B<\operatorname{deg} R$ such that $h=\frac{R_{<} \tilde{R}_{<}}{P_{<} \tilde{P}_{<}} h_{0}+\frac{B}{P}$. Hence $|h| \geq|| \frac{R_{<} \tilde{R}_{<}}{P_{<} \tilde{P}_{<}}|| h_{0}|-| \frac{B}{P}||$ with $m:=\operatorname{deg} R-\operatorname{deg} P_{<}-\operatorname{deg} P_{>} \geq 1$ and $l:=\operatorname{deg} B-\operatorname{deg} P \leq m-1$. Choose $h_{0} \in H_{+}$satisfying $\left|h_{0}\right|=(1+|x|)^{-3 / 4}$. ( $h_{0}$ exists by (2.1) since square-integrable and $\ln (1+|x|) \leq \sqrt{2|x|}$.) Because of $m-\frac{3}{4}-l>0$ the right side tends to $\infty$ like $|x|^{m-3 / 4}$ for $|x| \rightarrow \infty$ contradicting $h \in L^{2}(\mathbb{R})$. 
To complete the proof of the implication $(2) \Rightarrow(3)$ it remains to treat the case that $\operatorname{deg} P \geq \operatorname{deg} Q$ and $P$ has a real zero. Proceeding as in the forgoing case, here one has $\operatorname{deg} R=\operatorname{deg} P$. Hence, assuming that $P_{H_{+}}\left(\frac{P}{R} H_{+}\right)$is closed, one has $m \geq 1$ and $l \leq-1$ and the same contradiction follows.

From 3.2 one immediately obtains

Corollary 3.3 For $\lambda \in \mathbb{C}$ put $P^{\lambda}:=P+\lambda Q$. Then referring to $M_{+}(\kappa)$, $\lambda$ is

- a Fredholm point (i.e. $M_{+}(\kappa)-\lambda I$ is a Fredholm operator) iff $\lambda \notin \overline{\kappa(\mathbb{R})}$; if $\lambda$ is a Fredholm point, then $\operatorname{dim} \operatorname{ker}\left(M_{+}(\kappa)-\lambda I\right)=\max \left\{0, \operatorname{deg} Q_{>}-\operatorname{deg} P_{>}^{\lambda}\right\}$, $\operatorname{dim} \operatorname{ran}\left(M_{+}(\kappa)-\lambda I\right)^{\perp}=\max \left\{0, \operatorname{deg} P_{>}^{\lambda}-\operatorname{deg} Q_{>}\right\}$, and further $\operatorname{ind}\left(M_{+}(\kappa)-\right.$ $\lambda I)=\operatorname{deg} Q_{>}-\operatorname{deg} P_{>}^{\lambda}$

- a regular value (i.e. $M_{+}(\kappa)-\lambda I$ is continuously invertible) iff $\lambda \notin \overline{\kappa(\mathbb{R})}$ and $\operatorname{deg} Q_{>} \leq \operatorname{deg} P_{>}^{\lambda}$

- in the resolvent set iff $\lambda \notin \overline{\kappa(\mathbb{R})}$ and $\operatorname{deg} Q_{>}=\operatorname{deg} P_{>}^{\lambda}$

- a spectral value iff $\lambda \in \overline{\kappa(\mathbb{R})}$ or $\operatorname{deg} Q_{>} \neq \operatorname{deg} P_{>}^{\lambda}$

- in the point spectrum iff $\operatorname{deg} Q_{\leq}<\operatorname{deg} P_{<}^{\lambda}$ and $\operatorname{deg} P_{\geq}^{\lambda}<\operatorname{deg} Q_{>}$

- in the continuous spectrum (i.e. $M_{+}(\kappa)-\lambda I$ is injective with dense not closed range) iff $\lambda \in \overline{\kappa(\mathbb{R})}$, deg $P_{>}^{\lambda} \leq \operatorname{deg} Q_{>}$and either $\operatorname{deg} P_{<}^{\lambda} \leq \operatorname{deg} Q_{\leq}$or $\operatorname{deg} Q_{>} \leq$ $\operatorname{deg} P_{\geq}^{\lambda}$

- in the residual spectrum (i.e. $M_{+}(\kappa)-\lambda I$ is injective with not dense range) iff $\operatorname{deg} Q_{>}<\operatorname{deg} P_{>}^{\lambda}$

The characterization of the Fredholm points of $M_{+}(\kappa)$ and the fact that at a Fredholm point either the kernel or the deficiency space is trivial, are familiar from Krein's theory [3] for the case of integrable kernel. $\lambda \mapsto \operatorname{deg} P_{>}^{\lambda}$ is locally non-decreasing due to the continuity of the roots of a polynomial on its coefficients [26]. On $\mathbb{C} \backslash \overline{\kappa(\mathbb{R})}$ it is even locally constant, since there $P_{>}^{\lambda}=P_{\geq}^{\lambda}$. Hence, besides ind $\left(M_{+}(\kappa)-\lambda I\right)$, also $\operatorname{dim} \operatorname{ker}\left(M_{+}(\kappa)-\lambda I\right)$ and $\operatorname{dim} \operatorname{ran}\left(M_{+}(\kappa)-\lambda I\right)^{\perp}$ are constant on the components of $\mathbb{C} \backslash \overline{\kappa(\mathbb{R})}$.

Theorem 3.4 Let $\kappa$ be a real rational function. Let $p, q$ be real polynomials without common zeros such that $\kappa=\frac{p}{q}$ and put

$$
p-\mathrm{i} q=Q_{+} Q_{-}
$$

where the zeros of the polynomials $Q_{+}$and $Q_{-}$are all in the upper and lower halfplane, respectively. Then $M_{+}(\kappa)$ is closed symmetric with $\operatorname{dom} M_{+}(\kappa)=\frac{q^{\prime}}{(x+\mathrm{i}){ }^{5}} H_{+}$. By definition the zeros of $q^{\prime}$ are the real zeros of $q$ and $\varsigma:=\max \left\{\operatorname{deg} q^{\prime}, \operatorname{deg} p-\right.$ $\left.\operatorname{deg} q+\operatorname{deg} q^{\prime}\right\}$. The deficiency indices are

$$
n_{ \pm}:=\operatorname{dim} \operatorname{ran}\left(M_{+}(\kappa) \mp \mathrm{i} I\right)^{\perp}=\operatorname{deg} Q_{ \pm}-\operatorname{deg} q_{<}
$$


and the deficiency spaces are

$$
\begin{aligned}
& \operatorname{ran}\left(M_{+}(\kappa)-\mathrm{i} I\right)^{\perp}=\left\{\frac{q_{<}}{\widetilde{Q}_{+}} r: r \text { polynomial with } \operatorname{deg} r<\operatorname{deg} Q_{+}-\operatorname{deg} q_{<}\right\} \\
& \operatorname{ran}\left(M_{+}(\kappa)+\mathrm{i} I\right)^{\perp}=\left\{\frac{q_{<}}{Q_{-}} r: \text { r polynomial with } \operatorname{deg} r<\operatorname{deg} Q_{-}-\operatorname{deg} q_{<}\right\}
\end{aligned}
$$

Proof For dom $M_{+}(\kappa)$ see 3.2(a). Adopting the notation of 3.2 one has $M_{+}(\kappa)-$ i $I=$ $M_{+}\left(\frac{P}{Q}\right)$ with $P:=p-\mathrm{i} q$ and $Q=q$. Obviously $P$ and $Q$ have no common zeros. In view of $3.2(\mathrm{c})$ note $\tilde{Q}_{<}=q_{<}, \tilde{P}_{<}=\widetilde{Q}_{+}, \operatorname{deg} P_{>}=\operatorname{deg} Q_{+}, \operatorname{deg} Q_{>}=$ $\operatorname{deg} q_{>}=\operatorname{deg} q_{<}$. Hence the formula for $\operatorname{ran}\left(M_{+}(\kappa)-\mathrm{i} I\right)^{\perp}$ holds, which implies $n_{+}=\max \left\{0, \operatorname{deg} Q_{+}-\operatorname{deg} q_{<}\right\}$. It remains to show $\operatorname{deg} q_{<} \leq \operatorname{deg} Q_{+}$. The assertions about the deficiency space for $-\mathrm{i}$ follow similarly.

Let $m_{t}$ denote the number of zeros in the upper half-plane of $p-\mathrm{i} t q$ for $t>0$. $p-\mathrm{i} t q$ has no real zeros. So by the continuity of the roots of a polynomial, $m_{t}$ is locally constant and hence constant $=\operatorname{deg} Q_{+}$. Obviously $m_{t}$ is also the number of zeros in the upper half-plane of $\frac{1}{t} p-\mathrm{i} q$. Then by the same continuity the zeros of $q$ in the upper half-plane stay there for all $t$ large enough, whence $m_{t} \geq \operatorname{deg} q_{>}$. This yields the result.

$W_{\kappa}$ for $\kappa$ in 3.4 has unequal deficiency indices and hence no self-adjoint extension if $\max \{\operatorname{deg} p, \operatorname{deg} q\}$ is odd. The deficiency indices $\left(n_{+}, n_{-}\right)$of $W\left(x^{l}\right), l \in \mathbb{Z}$, are $\left(\frac{|l|}{2}, \frac{|l|}{2}\right)$ if $l$ is even and $\left(\frac{|l+1|}{2}, \frac{|l-1|}{2}\right)$ otherwise. Other interesting examples are $W\left(\frac{x^{2}+1}{x}\right)$ and $W\left(\frac{x^{2}-1}{x}\right)$ with deficiency indices $(1,1)$ and $(2,0)$, respectively. Compare the later with $W\left(\frac{x^{2}-1}{x-2}\right)$ having deficiency indices $(1,1)$. - This section is concluded by a much needed

Example 3.5 There are WH, even essentially self-adjoint semibounded ones, which are not closed, as for instance $W_{|x|}\left(\right.$ or $\left.W_{1 /|x|}\right)$.

As to the proof, by 3.2(a), dom $M_{+}(|x|)=\frac{1}{x+\mathrm{i}} H_{+}$and hence $R_{ \pm}:=\operatorname{ran}\left(M_{+}(|x|) \mp\right.$ $\mathrm{i} I)=\frac{|x| \mp \mathrm{i}}{x+\mathrm{i}} H_{+}$. It suffices to show that $R_{+}$is dense and $\neq H_{+}$.

The latter is easily inferred. Assume the contrary. Then there is $h \in H_{+}$satisfying $\frac{|x|-\mathrm{i}}{x+\mathrm{i}} h=\frac{1}{x+\mathrm{i}}$. Hence $h=\frac{1}{|x|-\mathrm{i}} \in H_{+}$and $h=\breve{h} \in H_{-}$, whence the contradiction $h=0$.

As to the former claim, let $h_{ \pm} \in R_{ \pm}^{\perp}$. Then $\left\langle h_{ \pm}, \frac{|x| \mp \mathrm{i}}{x+\mathrm{i}} h\right\rangle=0$ for all $h \in H_{+}$, whence $k_{ \pm}:=\frac{|x| \pm \mathrm{i}}{x-\mathrm{i}} h_{ \pm} \in H_{-}$. Let $\phi_{ \pm}$be holomorphic on the upper half-plane with $\phi_{ \pm, y} \rightarrow h_{ \pm}$for $0<y \rightarrow 0$ pointwise a.e. and in the mean (cf. 2.1). Similarly let $\psi_{ \pm}$ be holomorphic in the lower half-plane with $\psi_{ \pm, y} \rightarrow k_{ \pm}$for $0>y \rightarrow 0$. Consider $\phi:=(z+\mathrm{i}) \phi_{-} \phi_{+}$and $\psi:=(z-\mathrm{i}) \psi_{-} \psi_{+}$holomorphic on the upper and lower halfplane, respectively. Check that $\phi_{y}$ for $0<y \rightarrow 0$ and $\psi_{y}$ for $0>y \rightarrow 0$ converge pointwise a.e. and in $L_{l o c}^{1}$ to $f:=(x+\mathrm{i}) h_{-} h_{+}$. By [23, Theorem II] there is an entire function $\chi$ extending $\phi$ and $\psi$. Let $\chi_{ \pm}$be equal to $\phi_{ \pm}$and $\psi_{ \pm}$on the upper and lower half-plane, respectively. Let $z=x+\mathrm{i} y,|z|>1$. Then $\pi|\chi(z)| \leq \int_{|w| \leq 1} \mid \chi(z+$ $w) \mid \mathrm{d}^{2} w \leq(|z|+2)\left(\int_{|w| \leq 1}\left|\chi_{+}(z+w)\right|^{2} \mathrm{~d}^{2} w \int_{|w| \leq 1}\left|\chi_{-}(z+w)\right|^{2} \mathrm{~d}^{2} w\right)^{1 / 2}$. Now 
$\int_{|w| \leq 1}\left|\chi_{ \pm}(z+w)\right|^{2} \mathrm{~d}^{2} w \leq \int_{-1}^{1} \int_{-\infty}^{\infty}\left|\chi_{ \pm}(u+\mathrm{i}(y+v))\right|^{2} \mathrm{~d} u \mathrm{~d} v \leq \int_{-1}^{1} C \mathrm{~d} v=2 C$ for some finite constant $C$. Hence $|\chi(z)| \leq C^{\prime}|z| \forall|z|>1$. So $\chi$ is a polynomial $a+b z$. For $y>0, x \mapsto \phi_{-, y}(x) \phi_{+, y}(x)=\frac{a+b z}{z+\mathrm{i}}$ is integrable on $\mathbb{R}$. This implies $a=b=0$ and hence $\chi=0$. Therefore either $h_{+}=0$ or $h_{-}=0$. This means that one and hence both deficiency spaces are $\{0\}$. So $R_{+}$is dense.

\section{Semibounded Wiener-Hopf operators}

In 4.3, 4.4 a densely defined semibounded $\mathrm{WH} W_{\kappa}$ is expressed in a canonical way by the product of a closable operator and its adjoint. Replacing the operator by its closure one obtains a self-adjoint extension $\tilde{W}_{\kappa}$ of $W_{\kappa}$, which is semibounded by the same bound. The bound is not an eigenvalue of the extension. $\tilde{W}_{\kappa}$ is shown to be the Friedrichs extension of $W_{\kappa}$. We start with a preparatory lemma. The condition $\gamma \neq 0$ not a.e. means that $\gamma^{-1}(\{0\})$ is not a null set.

Lemma 4.1 Let $\gamma: \mathbb{R} \rightarrow \mathbb{C}$ be measurable. Put $E:=\gamma^{-1}(\mathbb{C} \backslash\{0\})$ and let $A:=$ $P_{+} \mathcal{F} M(\gamma) P_{E}^{*}$. Then $A$ is densely defined and $A^{*}=P_{E} M(\bar{\gamma}) \mathcal{F}^{-1} P_{+}^{*}$ holds, and $\operatorname{dom} A^{*}=\operatorname{dom} W_{\bar{\gamma}}$ is either $\{0\}$ or dense. If $\gamma$ is not almost zero, then $A^{*}$ is injective. If $\gamma \neq 0$ not a.e., then $A$ is injective. If $\gamma \neq 0$ a.e., then $\operatorname{ker} A=\left\{f \in L^{2}(\mathbb{R})\right.$ : $\left.\gamma f \in H_{-}\right\}$, which equals $\{0\}$ if and only if $\frac{1}{\gamma}$ is not proper. Finally $\operatorname{ker} A^{*} A=\operatorname{ker} A$, $W\left(|\gamma|^{2}\right)=A A^{*}$, and

$$
\gamma \text { proper } \Leftrightarrow \operatorname{dom} W\left(|\gamma|^{2}\right) \neq\{0\} \Leftrightarrow \operatorname{dom} A^{*} \neq\{0\} \Leftrightarrow \text { A closable }
$$

Proof Note $M(\gamma) P_{E}^{*}=P_{E}^{*} M\left(\left.\gamma\right|_{E}\right)$ and $P_{E} M(\gamma)=M\left(\left.\gamma\right|_{E}\right) P_{E}$ with $M\left(\left.\gamma\right|_{E}\right)$ the multiplication operator in $L^{2}(E)$. Hence $A=P_{+} \mathcal{F} P_{E}^{*} M\left(\left.\gamma\right|_{E}\right)$ is densely defined, and $A^{*}=P_{E} M(\bar{\gamma}) \mathcal{F}^{-1} P_{+}^{*}$ by $[27,13.2(2)]$. Note that $\operatorname{dom} A^{*}=\operatorname{dom} M(\bar{\gamma}) \mathcal{F}^{-1} P_{+}^{*}=$ $\operatorname{dom} W_{\bar{\gamma}}$, whence the claim on $\operatorname{dom} A^{*}$ by 2.10 .

First let $E$ be proper. Then $A^{*} g=M\left(\left.\bar{\gamma}\right|_{E}\right)\left(P_{E} \mathcal{F}^{-1} P_{+}^{*} g\right)=0$ implies $P_{E} \mathcal{F}^{-1} P_{+}^{*} g=0$, whence $g=0$. Similarly, $A k=P_{+} \mathcal{F}\left(P_{E}^{*} M\left(\left.\gamma\right|_{E}\right) k\right)=0$ means that $f_{-}:=P_{E}^{*} M\left(\left.\gamma\right|_{E}\right) k \in H_{-}$and vanishes on $\mathbb{R} \backslash E$, whence $f_{-}=0$ and hence $k=0$. - Now assume at once $E=\mathbb{R}$. Then $A^{*}=M(\bar{\gamma}) \mathcal{F}^{-1} P_{+}^{*}$ is injective as $M(\bar{\gamma})$ is injective. Furthermore, $f \in \operatorname{ker} A \Leftrightarrow f, \gamma f \in L^{2}(\mathbb{R})$ with $P_{+} \mathcal{F}(\gamma f)=0 \Leftrightarrow f \in L^{2}(\mathbb{R}), \gamma f \in H_{-} \Leftrightarrow f \in L^{2}(\mathbb{R}), \bar{f} \in \frac{1}{\bar{\gamma}} H_{+}$, and recall that $\frac{1}{\gamma}$ is proper $\Leftrightarrow \frac{1}{\bar{\gamma}} h \in L^{2}(\mathbb{R})$ for some $h \in H_{+} \backslash\{0\}$.

$\operatorname{ker} A^{*} A=\operatorname{ker} A$ is obvious since either $\gamma=0$ a.e. or $A^{*}$ is injective. $M(\gamma) P_{E}^{*} P_{E} M(\bar{\gamma})=M(\gamma) M\left(1_{E}\right) M(\bar{\gamma})=M\left(|\gamma|^{2}\right)$, whence $A A^{*}=W\left(|\gamma|^{2}\right)$.

Turn to the final claim $(\star)$. Recall that $\operatorname{dom} A^{*}=\operatorname{dom} W_{\bar{\gamma}}$ is either trivial or dense. So the last equivalence is standard and the remaining equivalences hold by 2.7 .

In view of $4.1(\star)$ recall the results on the domain of a WH in Sect. 2. If $A$ is closable it need not be closed, even if $A A^{*}$ is closed. (Indeed, $W_{x^{2}}=A A^{*}$ is closed and $\neq \tilde{W}_{x^{2}}=\bar{A} A^{*}$ by $3.4,4.3$.) Recall that ran $A^{*}$ is not dense if $A$ is not injective. $A$ is not injective for $\gamma \neq 0$ a.e. if for instance $\frac{q}{\gamma} \mathcal{F}^{-1} s \in L^{2}(\mathbb{R})$ with $q$ a polynomial and $s$ 
a Schwartz function with support in ] $-\infty, 0]$. (Indeed, $q \mathcal{F}^{-1} s$ is a Schwartz function in $H_{-}$, whence $\frac{q}{\gamma} \mathcal{F}^{-1} s \in \operatorname{ker} A$.) Recall that $A$ is injective if and only if $\gamma \neq 0$ not a.e. or $\gamma \neq 0$ a.e. and $\frac{1}{\gamma}$ not proper.

Lemma 4.2 Let $\gamma$ be proper. Suppose that $\gamma \neq 0$ not a.e. or that $\gamma \neq 0$ a.e. and $\frac{1}{\gamma}$ is not proper. Then $A$ is closable and $\bar{A}$ is injective.

Proof $A$ is closable by 4.1( $\star$ ). According to $2.6(p=\infty)$ one has $|\gamma| \mathrm{e}^{j} \leq 1$ for some $j$. Put $j^{\prime}:=1_{\{j \leq 0\}} j-|x|^{1 / 2}$. Then $|\gamma| \mathrm{e}^{j^{\prime}} \leq 1$, and $\mathrm{e}^{j^{\prime}}$ and $|\gamma| \mathrm{e}^{j^{\prime}}$ are square integrable. By (2.1) there is $h \in H_{+}$with $|h|=\mathrm{e}^{j^{\prime}}$. Hence $h \in \operatorname{dom} M_{+}(\bar{\gamma}) \backslash\{0\}$. Then $h D \subset$ $\operatorname{dom} M_{+}(\bar{\gamma})$ for $D:=\frac{1}{x+\mathrm{i}} H_{+}^{\infty} . D$ is dense in $H_{+}$since $D=\Gamma\left(H^{\infty}(\mathbb{T})\right)$ (see 2.2). -Now let $f \in \operatorname{ker} \bar{A}=\left(\operatorname{ran} A^{*}\right)^{\perp}$. For $d \in D$ one has $0=\left\langle f, P_{E} M(\bar{\gamma}) P_{H_{+}}^{*}(h d)\right\rangle=$ $\left\langle P_{E}^{*} f, \bar{\gamma} h d\right\rangle=\left\langle\gamma \bar{h} P_{E}^{*} f, d\right\rangle$, whence $\gamma \bar{h} P_{E}^{*} f \in H_{-}$. If $\gamma \neq 0$ not a.e., then it follows $\gamma \bar{h} P_{E}^{*} f=0$, whence $f=0$. If $\gamma \neq 0$ a.e., then $h \bar{f}=\frac{1}{\bar{\gamma}} h^{\prime}$ for some $h^{\prime} \in H_{+}$. Assume $f \neq 0$. Then $h^{\prime} \neq 0$ and $|\gamma|^{-1}|| h^{\prime} \mid$ is integrable. Hence $|\gamma|^{-1}$ is proper by (2.1) and 2.6 $(p=1)$, which however is excluded by the premise.

The foregoing lemma is needed only in Sect. 5. The main result of this section follows.

Theorem 4.3 Let $\kappa \geq 0$. Put $E:=\kappa^{-1}(\mathbb{R} \backslash\{0\})$ and $A:=P_{+} \mathcal{F} M(\sqrt{\kappa}) P_{E}^{*}$. Then $W_{\kappa}=A A^{*}$. -Now let $\kappa$ be proper not almost zero. Put $\tilde{W}_{\kappa}:=\bar{A} A^{*}$. Then

(a) $W_{\kappa}$ is densely defined symmetric nonnegative and $\tilde{W}_{\kappa}$ is an injective nonnegative self-adjoint extension of $W_{\kappa}$.

(b) $\operatorname{dom} W_{\kappa}$ is a core of $A^{*}$ and $\operatorname{dom} A^{*} \cap \operatorname{ran}\left(I+W_{\kappa}\right)^{\perp}=\{0\}$ holds.

(c) $\tilde{W}_{\kappa}$ is the Friedrichs extension of $W_{\kappa}$.

Proof (a) Apply 4.1 for $\gamma:=\sqrt{\kappa}$. Accordingly, $W_{\kappa}=A A^{*}$ and, if $\kappa$ is proper, $W_{\kappa}$ is densely defined and symmetric nonnegative by $2.14,2.15$, and $A^{*}$ is densely defined. Then $\bar{A}=A^{* *}$ and by $[27,13.13(\mathrm{a})] \bar{A} A^{*}$ is self-adjoint. Clearly $\bar{A} A^{*}$ is nonnegative. Check that $\bar{A} A^{*}$ is injective as $A^{*}$ is injective by 4.1 for $\kappa$ not almost zero.

(b), (c) According to [4, Theorem X.23], $\tilde{W}_{\kappa}$ is the Friedrichs extension only if dom $\tilde{W}_{\kappa} \subset H_{W_{\kappa}}$, where $H_{W_{\kappa}}$ is the completion of dom $W_{\kappa}$ with respect to the sesquilinear form $\left\langle g, g^{\prime}\right\rangle_{W_{\kappa}}:=\left\langle g, g^{\prime}\right\rangle+\left\langle g, W_{\kappa} g^{\prime}\right\rangle$.

Endow dom $A^{*}$ with the inner product $\left\langle g, g^{\prime}\right\rangle_{A^{*}}:=\left\langle g, g^{\prime}\right\rangle+\left\langle A^{*} g, A^{*} g^{\prime}\right\rangle$, by which $\operatorname{dom} A^{*}$ becomes a Hilbert space $\mathcal{K}$ since $A^{*}$ is closed. Then the subspace $\operatorname{dom} \bar{A} A^{*}$ is dense in $\mathcal{K}$ since $\operatorname{dom} \bar{A} A^{*}$ is a core for $A^{*}$, see [27, 13.13(b)]. One easily checks that $H_{W_{\kappa}}$ is the closure of $\operatorname{dom} W_{\kappa}$ in $\mathcal{K}$. Therefore $\operatorname{dom} \bar{A} A^{*} \subset H_{W_{\kappa}}$ if and only if $H_{W_{\kappa}}=\mathcal{K}$, which means that dom $W_{\mathcal{K}}$ is a core of $A^{*}$. A short computation shows also that $H_{W_{\kappa}}=\mathcal{K}$ is equivalent to $\operatorname{dom} A^{*} \cap \operatorname{ran}\left(I+W_{\kappa}\right)^{\perp}=\{0\}$.

Hence it remains to show $\operatorname{dom} A^{*} \cap \operatorname{ran}\left(I+W_{\kappa}\right)^{\perp}=\{0\}$. Explicitly this means that $h_{0}=0$ if

$$
h_{0} \in H_{+}, \sqrt{\kappa} h_{0} \in L^{2}(\mathbb{R}),\left\langle h_{0},(1+\kappa) h\right\rangle=0 \forall h \in H_{+} \text {with } \kappa h \in L^{2}(\mathbb{R})
$$

To this end a sequence $\left(\alpha_{n}\right)_{n}$ in $H_{+}^{\infty}$ will be constructed with $\left|\alpha_{n}\right|=\mathrm{e}^{j_{n}}$ a.e., where $j_{n}(x):=0$ if $1+\kappa(x)<n$ and $j_{n}(x):=-\frac{1}{2} \ln (1+\kappa(x))$ otherwise, and 
satisfying $\alpha_{n_{k}} \rightarrow 1$ pointwise a.e. for some subsequence $\left(n_{k}\right)$. Provided $\left(\alpha_{n}\right)_{n}$ set $h_{n}:=\alpha_{n} h_{0}$. Then $h_{n} \in H_{+}$and almost everywhere $(1+\kappa(x))\left|h_{n}(x)\right|$ is less than $n\left|h_{0}(x)\right|$ if $1+\kappa(x) \leq n$ and equals $\sqrt{1+\kappa(x)}\left|h_{0}(x)\right|$ otherwise, which proves $(1+\kappa) h_{n} \in L^{2}(\mathbb{R})$. Moreover $\sqrt{1+\kappa}\left|h_{n}\right| \leq \sqrt{1+\kappa}\left|h_{0}\right|$ since $\left|\alpha_{n}\right| \leq 1$ and $\sqrt{1+\kappa} h_{n_{k}} \rightarrow \sqrt{1+\kappa} h_{0}$ pointwise a.e., whence $\sqrt{1+\kappa} h_{n_{k}} \rightarrow \sqrt{1+\kappa} h_{0}$ in $L^{2}(\mathbb{R})$ by dominated convergence. Thus $(\star)$ holds for $h=h_{n_{k}}$, whence $0=\left\langle h_{0},(1+\kappa) h_{n_{k}}\right\rangle=$ $\left\langle\sqrt{1+\kappa} h_{0}, \sqrt{1+\kappa} h_{n_{k}}\right\rangle \rightarrow\left\langle\sqrt{1+\kappa} h_{0}, \sqrt{1+\kappa} h_{0}\right\rangle=\left\|\sqrt{1+\kappa} h_{0}\right\|^{2}$ implying $h_{0}=0$.

We turn to the construction of $\left(\alpha_{n}\right)_{n}$. By $2.8, \frac{\ln (1+\kappa)}{1+x^{2}}$ and hence all $\frac{j_{n}}{1+x^{2}}$ are integrable. For convenience we pass from $\mathbb{R}$ to the torus $\mathbb{T}$ by means of the Cayley transformation $C$ (see 2.2). So let $\tilde{j}_{n}:=j_{n} \circ C^{-1}$, which is integrable on $\mathbb{T}$. Put

$$
F_{n}(w):=\frac{1}{2 \pi} \int_{0}^{2 \pi} \frac{\mathrm{e}^{\mathrm{i} t}+w}{\mathrm{e}^{\mathrm{i} t}-w} \tilde{j}_{n}\left(\mathrm{e}^{\mathrm{i} t}\right) \mathrm{d} t
$$

for $w \in \mathbb{D}$. Then $\exp \circ F_{n}$ is an outer function. Let $\tilde{\alpha}_{n}$ denote its non-tangential limit a.e. on $\mathbb{T}$. It satisfies $\left|\tilde{\alpha}_{n}\right|=\mathrm{e}^{\tilde{j}_{n}}$ a.e. Hence $\tilde{\alpha}_{n} \in H^{\infty}(\mathbb{T})$. It remains to show the existence of a subsequence $\left(n_{k}\right)$ satisfying $\tilde{\alpha}_{n_{k}} \rightarrow 1$ a.e. The formula $T f(z):=$ $\lim _{r \uparrow 1} \frac{1}{2 \pi} \int_{0}^{2 \pi} \frac{\mathrm{e}^{\mathrm{i} t}+r z}{\mathrm{e}^{\mathrm{i} t}-r z} f\left(\mathrm{e}^{\mathrm{i} t}\right) \mathrm{d} t, z \in \mathbb{T}$ defines a bounded operator on $L^{1}(\mathbb{T})$ into weak- $L^{1}(\mathbb{T})$, whence $|\{z \in \mathbb{T}:|T f|>\delta\}| \leq C\|f\|_{1} / \delta$ for all $\delta>0$ and $f \in L^{1}(\mathbb{T})$. Therefore, if $f_{n} \rightarrow 0$ in $L^{1}(\mathbb{T})$, then $T f_{n} \rightarrow 0$ in probability, which implies $T f_{n_{k}} \rightarrow 0$ a.e. for some subsequence $\left(n_{k}\right)$. This applies to $\left(\tilde{j}_{n}\right)_{n}$ yielding $\tilde{\alpha}_{n_{k}}=\exp \circ T \tilde{j}_{n_{k}} \rightarrow 1$ a.e.

For $\kappa \geq 0$ and $W_{\kappa}$ densely defined recall that the deficiency subspace $\operatorname{ran}\left(I+W_{\kappa}\right)^{\perp}$ of $W_{\kappa}$ at -1 is trivial if and only if $W_{\kappa}$ is essentially self-adjoint.

Semibounded symbol 4.4 Let $\kappa$ be real semibounded. Then there are $\alpha>0$ and $\eta \in\{1,-1\}$ such that $\kappa^{\prime}:=\alpha 1+\eta \kappa \geq 0$. Clearly $W_{\kappa^{\prime}}=\alpha I+\eta W_{\kappa}$. Let $W_{\kappa}$ be densely defined. Then so is $W_{\kappa^{\prime}}$, and according to 4.3 there is the injective nonnegative self-adjoint extension $\tilde{W}_{\kappa^{\prime}}$ of $W_{\kappa^{\prime}}$. So

$$
\tilde{W}_{\kappa}:=-\eta \alpha I+\eta \tilde{W}_{\kappa^{\prime}}
$$

is a semibounded self-adjoint extension of $W_{\kappa}$ with bound $-\eta \alpha$, which is not an eigenvalue of $\tilde{W}_{\kappa}$. It is the Friedrichs extension of $W_{\kappa}$.

\section{Isomorphic Singular Integral Operators}

This section is concerned with the symmetric singular integral operator in $L^{2}(E)$ for proper $E$ or $E=\mathbb{R}$

$$
\left(L_{\phi} f\right)(x):=\frac{1}{2} \phi(x) f(x)+\frac{1}{2 \pi \mathrm{i}} \int_{E} \frac{\sqrt{\phi(x)} \sqrt{\phi(y)}}{y-x} f(y) \mathrm{d} y
$$


(in the sense of the principal value at $x$ ) where $\phi: E \rightarrow \mathbb{R}$ is measurable positive. $L_{\phi}$ will turn out to be closely related to $W_{\kappa}$, where $\kappa$ extends $\phi$ on $\mathbb{R}$ by zero.

$L_{\phi}$ is rather general. Indeed, it belongs to the studied class of singular integral operators in $L^{2}(E)$ of Hilbert transformation type

$$
(L(a, b) f)(x):=a(x) f(x)+\frac{1}{\mathrm{i} \pi} \int_{E} b(x) \overline{b(y)}(y-x)^{-1} f(y) \mathrm{d} y
$$

where $a, b$ are measurable functions on $E$ with $a$ real and $b \neq 0$ a.e. There is the obvious unitary equivalence

$$
U L(a, b) U^{-1}=L_{\phi}+M(\alpha)
$$

for $\phi=2|b|^{2}$ and $\alpha=a-|b|^{2}$, where $U$ is the multiplication operator by $\bar{b} /|b|$ and $M(\alpha)$ the multiplication operator by $\alpha$ in $L^{2}(E)$. So we are concerned with the case $a=|b|^{2}$.

The operator $L(a, b)$ for bounded $b$ and bounded below $a$ is treated by Rosenblum in [28]. It is shown to be self-adjoint on $\operatorname{dom} L(a, b)=\operatorname{dom} M(a)$, and its diagonalization is achieved. See also [29] and the literature cited in [28,29]. The really unbounded case however is there when $b$ is unbounded. [30] is concerned with this case replacing $L(a, b)$ by the limit of truncated $L\left(a_{n}, b_{n}\right)$ which are bounded. Our analysis of $L_{\phi}$ will show 5.3 that $L(a, b) \subset M(\alpha)$ if the extension of $b$ on $\mathbb{R}$ by zero is not proper. Hence for $L(a, b)$ in (5.2) being not trivial it is necessary that the extension of $b$ is proper. In this case $L_{\phi}$ in (5.3) has a self-adjoint extension 5.5.

The Hilbert transformation $H$ on $L^{2}(\mathbb{R})$ is defined by the singular integral

$$
H f(x)=\frac{1}{\mathrm{i} \pi} \int_{-\infty}^{\infty} \frac{f(y)}{y-x} \mathrm{~d} y
$$

Recall its representation

$$
H=\mathcal{F}^{-1} M(\operatorname{sgn}) \mathcal{F}=-\mathcal{F} M(\text { sgn }) \mathcal{F}^{-1}
$$

on $L^{2}(\mathbb{R})$ with sgn the signum function on $\mathbb{R}$ (see e.g. [14, Theorems 91,95] or [22, Lemma 1.35] or [15, Chapter II 4.3], and for more details [31, Teorema 1.1.1]). Let us introduce

$$
H_{E}:=P_{E} H P_{E}^{*}
$$

the trace on $L^{2}(E)$ of the Hilbert transformation $H$. Its spectrum is determined in [32]. For $E=[a, b],-\infty \leq a<b \leq \infty, H_{E}$ is called finite and semi-finite Hilbert transformation if $E$ is bounded and semi-bounded, respectively. Its spectral representation is achieved in [33]. 


\subsection{Isometry relating $\tilde{L}_{\phi}$ to $\tilde{W}_{\kappa}$}

In what follows we use the polar decomposition $C=S|C|$ of a closed densely defined operator $C$ from a Hilbert space $\mathcal{H}$ into another $\mathcal{H}^{\prime}$ (see e.g. [4, VIII.9]). $|C|$ denotes the square root of the self-adjoint nonnegative operator $C^{*} C$ in $\mathcal{H}$. One has $\operatorname{dom}|C|=$ $\operatorname{dom} C$ and $\operatorname{dom} C^{*} C$ is a core for $C$. $S$ is a partial isometry from $\mathcal{H}$ into $\mathcal{H}^{\prime}$. Its initial space $(\operatorname{ker} S)^{\perp}$ equals $\overline{\operatorname{ran} C^{*}}=\overline{\operatorname{ran}|C|}=\overline{\operatorname{ran}|C|^{2}}$. Similarly, its final space $\operatorname{ran} S$ equals $\overline{\operatorname{ran} C}=\overline{\operatorname{ran}\left|C^{*}\right|}=\overline{\operatorname{ran}\left|C^{*}\right|^{2}}$. The partial isometry $S^{*}$ satisfies $\left(\operatorname{ker} S^{*}\right)^{\perp}=$ $\operatorname{ran} S$ and $\operatorname{ran} S^{*}=(\operatorname{ker} S)^{\perp}$. Note the important relation

$$
C C^{*}=S C^{*} C S^{*}
$$

which means that the reductions of $C C^{*}$ and $C^{*} C$ on the orthogonal complements of their respective null spaces are Hilbert space isomorphic by the restriction of the partial isometry $S$ to its initial state $(\operatorname{ker} S)^{\perp}$ and its final state $\operatorname{ran} S$.

Throughout this section $\kappa \geq 0$ and $\phi>0$ are related to each other by

$$
\phi=\left.\kappa\right|_{E} \text { for } E=\kappa^{-1}\left(\mathbb{R}_{+}\right)
$$

Lemma 5.1 Let $\kappa \geq 0, A=P_{+} \mathcal{F} M(\sqrt{\kappa}) P_{E}^{*}, A^{*}=P_{E} M(\sqrt{\kappa}) \mathcal{F}^{-1} P_{+}^{*}$. Then

(a) $W_{\kappa}=A A^{*}$

(b) $L_{\phi}=\frac{1}{2} M(\phi)+\frac{1}{2} M(\sqrt{\phi}) H_{E} M(\sqrt{\phi})=P_{E} M(\sqrt{\kappa}) P_{H_{+}}^{*} P_{H_{+}} M(\sqrt{\kappa}) P_{E}^{*}=A^{*} A$

Now suppose that $\kappa$ is proper. Let $\bar{A}=T|\bar{A}|$ be the polar decomposition of $\bar{A}$. Then the partial isometry $T: L^{2}(E) \rightarrow L^{2}\left(\mathbb{R}_{+}\right)$is surjective, its adjoint $T^{*}$ is injective, and

(c) $\tilde{W}_{\kappa}:=\bar{A} A^{*}$ is a self-adjoint extension of $W_{\kappa}$.

(d) $\tilde{L}_{\phi}:=A^{*} \bar{A}$ is a self-adjoint extension of $L_{\phi}$.

(e) $\operatorname{ker} \tilde{L}_{\phi}=\operatorname{ker} \bar{A},(\operatorname{ker} T)^{\perp}=\left(\operatorname{ker} \tilde{L}_{\phi}\right)^{\perp}=\overline{\operatorname{ran} A^{*}}$.

Proof (a) See 4.3. (b) Check $A^{*} A=P_{E} M(\sqrt{\kappa}) \mathcal{F}^{-1} P_{+}^{*} P_{+} \mathcal{F} M(\sqrt{\kappa}) P_{E}^{*}=$ $P_{E} M(\sqrt{\kappa}) P_{H_{+}}^{*} P_{H_{+}} M(\sqrt{\kappa}) P_{E}^{*}=P_{E} M(\sqrt{\kappa}) \frac{1}{2}(I+H) M(\sqrt{\kappa}) P_{E}^{*}$ by (5.5). So $A^{*} A=L_{\phi}$ as $P_{E} M(\sqrt{\kappa})=M(\sqrt{\phi}) P_{E}, M(\sqrt{\kappa}) P_{E}^{*}=P_{E}^{*} M(\sqrt{\phi})$. Recall (5.6). (c) and (d) are obvious. - Finally, $T$ is surjective as $\operatorname{ran} T=(\operatorname{ran} \bar{A})^{-}=\left(\operatorname{ker} A^{*}\right)^{\perp}=$ $L^{2}\left(\mathbb{R}_{+}\right)$by 4.1 , and (e) follows directly from polar decomposition.

As a first result we note that $L_{\phi}$ in (5.1) can be trivial:

Corollary 5.2 Let $\kappa \geq 0$ be not proper. Then $L_{\phi} \subset 0$. Moreover, dom $L_{\phi}=\{0\}$ if and only if either $\kappa \neq 0$ not a.e. or otherwise $\frac{1}{\kappa}$ is not proper. If $\kappa \neq 0$ a.e. and $\frac{1}{\kappa}$ is proper, then $\operatorname{dom} L_{\phi}=\frac{1}{\sqrt{\phi}} H_{-} \cap L^{2}(\mathbb{R})$.

Proof $\operatorname{dom} A^{*}=\{0\}$ by $4.1(\star)$, whence $L_{\phi} \subset 0$ and $\operatorname{dom} L_{\phi}=\operatorname{ker} A$ because of 5.1 (b), 4.1. Recall 2.7 by which $1 / \kappa$ is not proper if and only if $1 / \sqrt{\kappa}$ is not proper. The remainder is shown in 4.1 . 
For example $\kappa=\mathrm{e}^{x}$ is positive and, by $2.9, \kappa$ and $1 / \kappa$ are not proper. Hence dom $L_{\mathrm{e}^{x}}=$ $\{0\}$.

Now 5.2 and (5.3) imply that for $L(a, b)$ in (5.2) being not trivial it is necessary that the extension of $b$ on $\mathbb{R}$ by zero be proper:

Theorem 5.3 Let the extension of $b$ on $\mathbb{R}$ by zero be not proper. Then $L(a, b) \subset$ $M\left(a-|b|^{2}\right)$ with

(a) $\operatorname{dom} L(a, b)=\left\{f \in L^{2}(\mathbb{R}):|b| f \in H_{-},\left(a-|b|^{2}\right) f \in L^{2}(\mathbb{R})\right\}$ if $E=\mathbb{R}$

(b) $\operatorname{dom} L(a, b)=\{0\}$ if and only if $E$ is proper, or $E=\mathbb{R}$ and $\frac{1}{b}$ is not proper, or $E=\mathbb{R}$ and $\frac{1}{b}$ is proper and $\frac{a-|b|^{2}}{b}$ is not proper.

Corollary 5.4 Let $E=\mathbb{R}$ and suppose that $\frac{1}{\phi}$ is bounded.. Then $L_{\phi}$ is densely defined and

$$
\operatorname{dom} L_{\phi}=\left\{\frac{h+k}{\sqrt{\phi}}: h \in H_{+}, \sqrt{\phi} h \in L^{2}(\mathbb{R}), k \in H_{-}\right\}, L_{\phi}\left(\frac{h+k}{\sqrt{\phi}}\right)=\sqrt{\phi} h
$$

with $\operatorname{ker} L_{\phi}=\frac{1}{\sqrt{\phi}} H_{-}$and $\left(\operatorname{ker} L_{\phi}\right)^{\perp}=\left\{\sqrt{\phi} h \in L^{2}(\mathbb{R}): h \in H_{+}\right\}$. -If $\phi$ is proper, then $\operatorname{ker} \tilde{L}_{\phi}=\overline{\operatorname{ker} L_{\phi}} \neq L^{2}(\mathbb{R})$. If $\phi$ is not proper, then $\bar{L}_{\phi}=0$.

Proof Note $\kappa=\phi, L_{\phi}=M(\sqrt{\phi}) P_{H_{+}}^{*} P_{H_{+}} M(\sqrt{\phi})$ by 5.1(b). So $(\star)$ is easily verified and $\operatorname{ker} L_{\phi}=\frac{1}{\sqrt{\phi}} H_{-}$follows. Check $f \in L^{2}(\mathbb{R}), f \perp \frac{1}{\sqrt{\phi}} H_{-} \Leftrightarrow f \in L^{2}(\mathbb{R})$, $0=\left\langle f, \frac{1}{\sqrt{\phi}} k\right\rangle=\left\langle\frac{1}{\sqrt{\phi}} f, k\right\rangle \forall k \in H_{-} \Leftrightarrow f \in L^{2}(\mathbb{R}), \frac{1}{\sqrt{\phi}} f \in H_{+}$, proving the claim on $\left(\operatorname{ker} L_{\phi}\right)^{\perp}$.

Note $D:=\operatorname{dom} A^{*}=\left\{h \in H_{+}: \sqrt{\phi} h \in L^{2}(\mathbb{R})\right\}$, whence $\operatorname{ran} A^{*}=\{\sqrt{\phi} h \in$ $\left.L^{2}(\mathbb{R}): h \in H_{+}\right\}$which equals $\left(\operatorname{ker} L_{\phi}\right)^{\perp}$.

Now let $\phi$ be proper. Then $D$ is dense by 4.1 and $\operatorname{ran} A^{*} \neq\{0\}$. Hence by 5.1(e) on has $\operatorname{ker} \tilde{L}_{\phi}=\left(\operatorname{ran} A^{*}\right)^{\perp}=\overline{\operatorname{ker} L_{\phi}} \neq L^{2}(\mathbb{R})$. Moreover, dom $L_{\phi}$ is dense, since $f \in\left(\operatorname{dom} L_{\phi}\right)^{\perp} \Rightarrow 0=\langle f,(h+k) / \sqrt{\phi}\rangle=\langle f / \sqrt{\phi},(h+k)\rangle \forall h \in D, k \in H_{-} \Rightarrow$ $f / \sqrt{\phi} \in H_{+},\langle f / \sqrt{\phi}, h\rangle \forall h \in D \Rightarrow f / \sqrt{\phi}=0 \Rightarrow f=0$. - If $\phi$ is not proper then $D=\{0\}$ by 4.1 , whence $\operatorname{dom} L_{\phi}=\frac{1}{\sqrt{\phi}} H_{-}$. The latter equals $\operatorname{ker} L_{\phi}$. Finally, $\left(\operatorname{ker} L_{\phi}\right)^{\perp}=\left\{\sqrt{\phi} h \in L^{2}(\mathbb{R}): h \in H_{+}\right\}=\{0\}$ since $D=\{0\}$.

For example, $L_{\mathrm{e}}|x| \subset 0$ with dense dom $L_{\mathrm{e}} \mathrm{e}^{|x|}=\mathrm{e}^{-|x| / 2} H_{-}$. The main outcome of this section is the following:

Theorem 5.5 Let $\kappa \geq 0$ be proper. Then

$$
\tilde{L}_{\phi}=T^{*} \tilde{W}_{\kappa} T
$$

Let $\tilde{L}_{\phi}^{\prime}$ and $T^{\prime}$ denote the reduction of $\tilde{L}_{\phi}$ and the restriction of $T$ on the orthogonal complement of the null space of $\tilde{L}_{\phi}$, respectively. Then $T^{\prime}$ is a Hilbert space isomorphism onto $L^{2}\left(\mathbb{R}_{+}\right)$satisfying $\tilde{L}_{\phi}^{\prime}=T^{\prime-1} \tilde{W}_{\kappa} T^{\prime}$.

Proof (5.8) holds by polar decomposition $\bar{A}=T|\bar{A}|$ (cf. (5.7)). By $5.1 T$ is surjective. So $T^{\prime}$ is a Hilbert space isomorphism by 5.1(e). Recall (5.8). 
Of course one has $\tilde{W}_{\kappa}=T \tilde{L}_{\phi} T^{*}$ as well. It allows to study $\tilde{W}_{\kappa}$ starting from $\tilde{L}_{\phi}$. For the still remarkable bounded case see (5.9). By 4.4 it is easy to extend 5.5 to semibounded symbols.

Corollary 5.6 Let $\frac{\kappa}{1+x^{2}}$ be integrable. Then $\tilde{L}_{\phi}^{\prime}$ is absolutely continuous.

Proof Recall that $\tilde{T}_{\kappa \circ \gamma}$ and hence $\tilde{W}_{\kappa}$ (see 2.2) is absolutely continuous by [9]. Apply 5.5 .

The special case of 5.6 that $\phi$ is bounded is treated in [28, Sect. 3] and [29, Theorem]. -We remind that for $\tilde{L}_{\phi}$ being injective it is necessary that $E$ is proper or that $1 / \kappa$ is not proper.

Corollary 5.7 Let $\kappa \geq 0$ be proper. Suppose that $E$ is proper or $1 / \kappa$ is not proper. Then $\tilde{L}_{\phi}$ is injective and $\bar{T}: L^{2}(E) \rightarrow L^{2}\left(\mathbb{R}_{+}\right)$is a Hilbert space isomorphism with

$$
\tilde{L}_{\phi}=T^{-1} \tilde{W}_{\kappa} T
$$

Proof $\bar{A}$ is injective by 4.2. Hence $\{0\}=\operatorname{ker} \tilde{L}_{\phi}=\operatorname{ker} T$ and $T$ is an isomorphism. Apply 5.5.

So it is worth noting that $\tilde{L}_{\phi}$ is absolutely continuous, if $\frac{\kappa}{1+x^{2}}$ is integrable and if $E$ is proper or $1 / \kappa$ is not proper.

Let $\kappa$ be bounded. Then $L_{\phi}, W_{\kappa}$ are bounded, $\tilde{L}_{\phi}=L_{\phi}, \tilde{W}_{\kappa}=W_{\kappa}$, and

$$
L_{\phi}=T^{*} W_{\kappa} T, \quad W_{\kappa}=T L_{\phi} T^{*}
$$

Moreover, if $E$ is proper or $1 / \kappa$ is not proper, then even $L_{\phi}^{\prime}=L_{\phi}$ by 5.7 , whence $L_{\phi}=T^{-1} W_{\kappa} T$. An example for the latter case is $L_{\mathrm{e}^{-|x|}}$, which is injective. Generally, if $\kappa>0$ does not decrease too rapidly (so that $1 / \kappa$ is proper) the kernel of $L_{\kappa}$ is not trivial. For an instructive example see Sect.5.2. The trivial example here is $\kappa=1_{\mathbb{R}}$. Note that $W\left(1_{\mathbb{R}}\right)=I_{L^{2}\left(\mathbb{R}_{+}\right)}$and $L_{1_{\mathbb{R}}}$ is the orthogonal projection on $H_{+}$, and $T^{\prime}: H_{+} \rightarrow L^{2}\left(\mathbb{R}_{+}\right), T^{\prime} h:=P_{+} \mathcal{F} h$. Examples for proper $E$ are the isomorphic pairs $W\left(1_{E}\right) \simeq \frac{1}{2}\left(I+H_{E}\right)$ with $H_{E}$ in (5.6), which we like to write as

$$
H_{E}=T^{-1} W_{21_{E}-1} T
$$

The case of the finite Hilbert transformation $H_{[-1,1]}$ is studied in detail in [24, Sect. 3.3], $[24,(3.20)]$.

In conclusion we remark on the spectral representations of $\tilde{W}_{\kappa}$ and $\tilde{L}_{\phi}$ in 5.5. By the spectral theorem in the multiplication operator version, $\tilde{W}_{\kappa}$ is Hilbert space isomorphic to the multiplication operator $M(\varphi)$ on $L_{\mu}^{2}(\mathbb{R})$ for some Borel-measurable positive $\varphi: \mathbb{R} \rightarrow \mathbb{R}$ and finite Borel measure $\mu$. Let $V: L_{\mu}^{2}(\mathbb{R}) \rightarrow L^{2}\left(\mathbb{R}_{+}\right)$be an isomorphism satisfying

$$
\tilde{W}_{\kappa}=V M(\varphi) V^{-1}
$$


The spectral measure for $\tilde{W}_{\kappa}$ is given by $E_{\tilde{W}_{\kappa}}(\Delta)=V M\left(1_{\varphi^{-1}(\Delta)}\right) V^{-1}$ for measurable $\Delta \subset \mathbb{R}$.

Corollary 5.8 Suppose (5.11). Then $W_{0}:=A^{*} V M\left(\frac{1}{\sqrt{\varphi}}\right)$ with $A^{*}$ in 5.1 is closable, its closure $W$ is the Hilbert space isometry $T^{*} V$ with $\operatorname{ran} W=\left(\operatorname{ker} \tilde{L}_{\phi}\right)^{\perp}$, by which $\tilde{L}_{\phi}=W M(\varphi) W^{*}$.

Proof By (5.11) and 5.1(a), $W_{0}=A^{*} V M\left(\frac{1}{\sqrt{\varphi}}\right) V^{-1} V=A^{*} \tilde{W}_{\kappa}{ }^{-1 / 2} V=$ $A^{*}\left|A^{*}\right|^{-1} V=\left.T^{*}\right|_{\operatorname{dom}\left(\left|A^{*}\right|^{-1}\right)} V$. For the last equality recall $A^{*}=T^{*}\left|A^{*}\right|$. Further note that $\operatorname{dom}\left(\left|A^{*}\right|^{-1}\right)=\operatorname{ran}\left(\left|A^{*}\right|\right)$ is dense as $\left|A^{*}\right|=\tilde{W}_{\kappa}{ }^{1 / 2}$ is self-adjoint injective. Since $T^{*}$ is bounded, $W_{0}$ is closable and its closure $W$ equals $T^{*} V$. The remainder is obvious.

\subsection{Example: Lalescu's Operator}

Supposing $\kappa>0, \kappa \in L^{2}$, and kernel $k \in L^{1}$, in [34] a spectral theory of $W_{\kappa}$ is proposed by a reduction to a previously developed theory for singular integral operators. Its application in [34] to Lalescu's operator $W_{\lambda}$ with symbol

$$
\lambda(x):=\frac{2}{1+x^{2}}
$$

however does not produce the right normalization (5.14) of the generalized eigenfunctions (5.13). In establishing (5.14) we get also the result 5.10 on orthogonal polynomials.

The diagonalization of Lalescu's operator $W_{\lambda}$ is achieved in Sect.5.2.1 and that of the associated singular integral operator $L_{\lambda}$ is derived in Sect. 5.2.2. The generalized eigenfunctions (5.18) of the latter are no longer regular distributions.

\subsubsection{Spectral Representation of $W_{\lambda}$}

Clearly, the spectrum of $W_{\lambda}$ lies in $[0,2]$. The integral kernel for $W_{\lambda}$ is $\mathrm{e}^{-|x|}$. The obvious ansatz $a \mathrm{e}^{\alpha x}+b \mathrm{e}^{\beta x}$ for $u(x), x>0$ in $s u(x)-\int_{0}^{\infty} \mathrm{e}^{-|x-y|} u(y) \mathrm{d} y=0$ yields for every $s \in] 0,2$ [ the generalized eigenfunction (cf. [2])

$$
q_{s}(x)=n(s)\left((\tau-\mathrm{i}) \mathrm{e}^{\mathrm{i} \tau x}+(\tau+\mathrm{i}) \mathrm{e}^{-\mathrm{i} \tau x}\right), \quad \tau:=\left(\frac{2}{s}-1\right)^{1 / 2}
$$

The claim is that there is the unique positive normalization constant

$$
n(s)=(4 \pi s \tau)^{-1 / 2}
$$

such that $q(s, x):=q_{s}(x)$ is the kernel for a Hilbert space isomorphism

$$
V: L^{2}(0,2) \rightarrow L^{2}\left(\mathbb{R}_{+}\right), \quad V h=1 \text {. i.m. } \int_{\downarrow 0}^{\uparrow 2} q(s, \cdot) h(s) \mathrm{d} s
$$


Indeed, the assertion follows immediately from the following Lemma 5.9 as $V=U^{*} \Gamma$ holds for the Hilbert space isomorphism

$$
\Gamma: L^{2}(0,2) \rightarrow L^{2}\left(\mathbb{R}_{+}\right), \quad \Gamma h(y):=\frac{2 \sqrt{y}}{1+y^{2}} h\left(\frac{2}{1+y^{2}}\right)
$$

due to the change of variable $\gamma(y):=\frac{2}{1+y^{2}}$.

Lemma $5.9 U g(x):=1$.i.m. $\int_{\downarrow 0}^{\uparrow \infty} u(x, y) g(y) \mathrm{d} y$ with

$$
u(x, y)=(2 \pi)^{-1 / 2}\left(1+x^{2}\right)^{-1 / 2}\left((x-\mathrm{i}) \mathrm{e}^{\mathrm{i} x y}+(x+\mathrm{i}) \mathrm{e}^{-\mathrm{i} x y}\right)
$$

is unitary on $L^{2}\left(\mathbb{R}_{+}\right)$.

Proof Using the generating function $l_{q}(x)=(1-q)^{-1} \exp \left(-\frac{1}{2} \frac{1+q}{1-q} x\right),|q|<1$ of the Laguerre functions $l_{n}, n \geq 0$ one finds $\lambda_{n}(x):=U l_{n}(x)=\sqrt{\frac{2}{\pi}}(1+$ $\left.x^{2}\right)^{-1 / 2}\left(\frac{x+\mathrm{i}}{1+2 \mathrm{i} x} \gamma(x)^{n}+\frac{x-\mathrm{i}}{1-2 \mathrm{i} x} \gamma(x)^{-n}\right)$ with $\gamma(x):=\frac{-1+2 \mathrm{i} x}{1+2 \mathrm{i} x}$. Note that $\int_{-\infty}^{\infty}(1+$ $\left.x^{2}\right)^{-1}\left(\frac{x+\mathrm{i}}{1+2 \mathrm{i} x}\right)^{2} \gamma(x)^{k} \mathrm{~d} x=0$ for $k \in \mathbb{Z}$, since the integral is a parametrization of the complex integration of $\frac{i}{4} \frac{3 z-i}{3-z} z^{k}$ along the unit circle. Using this it follows easily that $\lambda_{n}, n=0,1,2, \ldots$ are orthonormal in $L^{2}\left(\mathbb{R}_{+}\right)$. It remains to show the completeness of $\left(\lambda_{n}\right)_{n}$.

Let $\varphi \in] 0, \pi$ [ satisfy $\mathrm{e}^{\mathrm{i} \varphi}=\gamma(x), x>0$. Put $\xi:=\cos \varphi$. Then $\lambda_{n}(x)=$ $\sqrt{\frac{18(1-\xi)\left(1-\xi^{2}\right)}{\pi(5-3 \xi)}}\left(\frac{\sin (n+1) \varphi}{\sin \varphi}-\frac{1}{3} \frac{\sin n \varphi}{\sin \varphi}\right)=\sqrt{\frac{18(1-\xi)\left(1-\xi^{2}\right)}{\pi(5-3 \xi)}}\left(U_{n}(\xi)-\frac{1}{3} U_{n-1}(\xi)\right)$, where $U_{n}$ denotes the Chebyshev polynomial of the second kind of degree $n$ for $n=0,1,2, \ldots$ and $U_{-1}:=0[35,10.11(2)]$. Therefore the Hilbert space isomorphism $\Gamma: L^{2}\left(\mathbb{R}_{+}\right) \rightarrow$ $L^{2}(-1,1)$ due to the change of variable $\gamma(\xi):=\frac{1}{2} \sqrt{\frac{1+\xi}{1-\xi}}, \gamma^{\prime}(\xi)=\frac{1}{2} \sqrt{\frac{1-\xi}{1+\xi}}(1-\xi)^{-2}$ maps $\lambda_{n}$ onto $\Gamma \lambda_{n}(\xi)=\sqrt{\frac{9}{\pi(5-3 \xi)}}\left(1-\xi^{2}\right)^{1 / 4} Q_{n}(\xi)$, where $Q_{n}:=U_{n}-\frac{1}{3} U_{n-1}$ is a polynomial of degree $n$ for $n=0,1,2, \ldots$ Thus 5.10 is shown, which implies the assertion.

Corollary 5.10 Let $Q_{n}:=U_{n}-\frac{1}{3} U_{n-1}, n=0,1,2, \ldots$ with $U_{n}$ the Chebyshev polynomial of the second kind of degree $n$ and $U_{-1}:=0$. Then $\left(Q_{n}\right)_{n \geq 0}$ is a sequence of orthonormal polynomials on $[-1,1]$ with respect to the weight function $\xi \mapsto$ $\frac{9}{\pi} \frac{\sqrt{1-\xi^{2}}}{(5-3 \xi)}$. It obeys the recurrence $Q_{n+1}=2 \xi Q_{n}-Q_{n-1}$ for $Q_{0}=1, Q_{1}(\xi)=2 \xi-\frac{1}{3}$.

Theorem 5.11 The spectral representation $W_{\lambda}=V M\left(\mathrm{id}_{[0,2]}\right) V^{-1}$ holds.

Proof First note $|q(s, x)| \leq(s(2-s))^{-1 / 2}$. Let $h \in L^{2}(0,2)$ with support in $[\delta, 2-\delta]$ for some $0<\delta<1$. Then $\left(V M\left(\operatorname{id}_{[0,2]}\right) h\right)(x)=\int_{\delta}^{2-\delta} q(s, x) s h(s) \mathrm{d} s$. Substitute $s q(s, x)=\int_{0}^{\infty} \mathrm{e}^{-|x-y|} q(s, y) \mathrm{d} y$. The integrations can be interchanged by Fubini's theorem yielding $\int_{0}^{\infty} \mathrm{e}^{-|x-y|}\left(\int_{\delta}^{2-\delta} q(s, y) h(s) \mathrm{d} s\right) \mathrm{d} y=\int_{0}^{\infty} \mathrm{e}^{-|x-y|} V h(y) \mathrm{d} y=$ $W_{\lambda} V h(y)$. This implies the result. 


\subsubsection{Spectral Representation of $L_{\lambda}$}

Let us first establish the kernel of $L_{\lambda}$. By 5.1(e) the latter equals that of $A=$ $P_{+} \mathcal{F} M(\sqrt{\lambda})$, whence $\operatorname{ker}\left(L_{\lambda}\right)=\left\{f \in L^{2}(\mathbb{R}): \sqrt{\lambda} f \in H_{-}\right\}$by 4.1. Put $u(x):=\frac{x+\mathrm{i}}{\sqrt{1+x^{2}}}, x \in \mathbb{R}$. Note that the multiplication operator $M(u)$ is unitary. It follows that

$$
\operatorname{ker}\left(L_{\lambda}\right)=M(u) H_{-}, \quad \operatorname{ker}\left(L_{\lambda}\right)^{\perp}=M(u) H_{+}
$$

Indeed, as to the less trivial implication let $f \in \operatorname{ker}\left(L_{\lambda}\right)$. Then $h:=\sqrt{\lambda} f \in H_{-}$, whence $(x-\mathrm{i}) h \in L^{2}(\mathbb{R})$. Therefore $k:=2^{-1 / 2}(x-\mathrm{i}) h \in H_{-}$using 3.1(c), and $f=u k$ follows.

Hence the reduction $L_{\lambda}^{\prime}$ of $L_{\lambda}$ on the orthogonal complement of its kernel is self-adjoint bounded on $M(u) H_{+}$with spectrum [0,2]. The following computations are valid in a distributional sense. So generalized eigenfunctions $Q_{s}$ for $L_{\lambda}$ are given by $A^{*} q_{s}=M(\sqrt{\lambda}) \mathcal{F}^{-1} P_{+}^{*} q_{s}$ since $L_{\lambda} A^{*} q_{s}=A^{*} A A^{*} q_{s}=s A^{*} q_{s}$. Recall $\int_{-\infty}^{\infty} 1_{] 0, \infty[} \mathrm{e}^{\mathrm{i} x y} \mathrm{~d} y=\frac{\mathrm{i}}{x}+\pi \delta(x)=: \Delta(x)$, see e.g. [36, Table of Fourier Transforms $1.23]$. So one yields the not regular distributions on $\mathbb{R}$

$$
Q_{s}(x)=\frac{n(s)}{\sqrt{s}} \sqrt{\frac{2}{1+x^{2}}} \frac{1}{\sqrt{2 \pi}}((\tau-\mathrm{i}) \Delta(x+\tau)+(\tau+\mathrm{i}) \Delta(x-\tau))
$$

The claim is that $\frac{n(s)}{\sqrt{s}}$ is the unique positive normalization constant such that $Q(s, x):=$ $Q_{s}(x)$ is the kernel for an isometry $W$ on $L^{2}(0,2)$ in $L^{2}(\mathbb{R})$ satisfying

$$
W h=\int_{0}^{2} Q(s, \cdot) h(s) \mathrm{d} s
$$

for test functions $h$. The additional factor $\frac{1}{\sqrt{s}}$ regarding the normalization constant of $Q_{s}$ corresponds to the factor $\frac{1}{\sqrt{\varphi}}$ for $W_{0}$ in 5.8 and is suggested heuristically by $\left\langle A^{*} q_{s}, A^{*} q_{s}\right\rangle=\left\langle q_{s}, A A^{*} q_{s}\right\rangle=s\left\langle q_{s}, q_{s}\right\rangle$. For the proof recall the isomorphism $\Gamma$ (5.16) and the Hilbert transformation $H(5.5)$. For $g: \mathbb{R}_{+} \rightarrow \mathbb{C}$ let $g_{\text {oe }}$ denote the odd extension $g_{o e}(x)=-g(-x)$ for $x \leq 0$ of $g$.

Theorem 5.12 The integral operator (5.19) determines a Hilbert space isometry $W$ : $L^{2}(0,2) \rightarrow L^{2}(\mathbb{R})$ with $\mathrm{ran} W=M(u) H_{+}$. It satisfies $W h=M(u) \frac{1}{2}(I+H)(\Gamma h)_{o e}$ for $h \in L^{2}(0,2)$ and yields the representation

$$
L_{\lambda}=W M\left(\mathrm{id}_{[0,2]}\right) W^{*}
$$

Proof By the change of variable $t=2 /\left(1+s^{2}\right)$ for the integration in (5.19) one obtains $\sqrt{1+x^{2}} W h(x)=\frac{1}{2 \pi} \int_{0}^{\infty}((t-\mathrm{i}) \Delta(x+t)+(t+\mathrm{i}) \Delta(x-t)) \Gamma h(t) \mathrm{d} t$. The integral is easily done yielding $(\star) W h=M(u) \frac{1}{2}(I+H)(\Gamma h)_{o e}$ for test functions $h$. Check $\langle H f, f\rangle=0$ if $f \in L^{2}(\mathbb{R})$ is odd. Therefore $\|W h\|^{2}=\left\|\frac{1}{2}(I+H)(\Gamma h)_{o e}\right\|^{2}=$ 
$\left\langle\frac{1}{2}(I+H)(\Gamma h)_{o e},(\Gamma h)_{o e}\right\rangle=\frac{1}{2}\left\|(\Gamma h)_{o e}\right\|^{2}=\|h\|^{2}$. So $W$ is an isometry, and $(\star)$ extends to all $h \in L^{2}(0,2)$. Obviously $W h \in M(u) H_{+}$. Moreover, if $k \in H_{+}$then $k=\frac{1}{2}(I+H)(k-\breve{k})$ as $\breve{k} \in H_{-}$. Since $k-\breve{k}$ is odd, this implies ran $W=M(u) H_{+}$.

The spectral representation of $L_{\lambda}$ follows from $W M\left(\mathrm{id}_{[0,2]}\right) h=L_{\lambda} W h$ for test functions $h$. The latter is shown along the lines of the proof of 5.11. We omit the technicalities.

Funding Open Access funding enabled and organized by Projekt DEAL.

\section{Declarations}

Conflict of interest Not applicable.

Availability of data and material Not applicable.

Code availability Not applicable.

Open Access This article is licensed under a Creative Commons Attribution 4.0 International License, which permits use, sharing, adaptation, distribution and reproduction in any medium or format, as long as you give appropriate credit to the original author(s) and the source, provide a link to the Creative Commons licence, and indicate if changes were made. The images or other third party material in this article are included in the article's Creative Commons licence, unless indicated otherwise in a credit line to the material. If material is not included in the article's Creative Commons licence and your intended use is not permitted by statutory regulation or exceeds the permitted use, you will need to obtain permission directly from the copyright holder. To view a copy of this licence, visit http://creativecommons.org/licenses/by/4.0/.

\section{References}

1. Böttcher, A., Silbermann, B.: Analysis of Toeplitz Operators. Springer, Berlin (1990)

2. Wiener, N., Hopf, E.: Über eine Klasse singulärer Integralgleichungen, Sitz. Berlin. Akad. Wiss. pp. 696-706 (1931). See also [18, Chap. IV] and [14, 11.17]

3. Krein, M.G.: Integral equations on a half-line with kernels depending upon the difference of the arguments. Amer. Math. Soc. Transl. 2(22), 163-288 (1962)

4. Reed, M., Simon, B.: Methods of Modern Mathematical Physics. Academic Press, New York (1975)

5. Devinatz, A., Shinbrot, M.: General Wiener-Hopf Operators, Trans. AMS 145, 467-494 (1969). F. O. Speck: General Wiener-Hopf factorization methods, Pitman, Boston (1985)

6. Yafaev, D.R.: On Semibounded Wiener-Hopf Operators, J. London Math. Soc. 95, 742-762 (2017) or arXiv: 1606.01361v1 [math.FA]

7. Yafaev, D.R.: Toeplitz versus Hankel: Semibounded Operators. Opuscula Math. 38(4), 573-590 (2018). https://doi.org/10.7494/OpMath.2018.38.4.573

8. Rosenblum, M.: Self-adjoint Toeplitz operators and associated orthonormal functions. Proc. Am. Math. Soc. 13, 590-595 (1962)

9. Rosenblum, M.: A concrete spectral theory for self-adjoint Toeplitz operators. Am. J. Math. 87, 709_ 718 (1965)

10. Pellegrini, V.J.: Unbounded general Wiener-Hopf operators. Indiana Univ. Math. J. 21, 85-90 (1971)

11. Sarason, D.: Unbounded Toeplitz operators. Integr. Equ. Oper. Theory 61, 281-298 (2008)

12. Câmara, M.C., Malheiro, M.T., Partington, J.R.: Kernels of unbounded Toeplitz operators and factorization of symbols. Results Math. 76(1), 10 (2021)

13. Gustafson, K.: The Toeplitz-Hausdorff Theorem for Linear Operators. In Proceedings of the American Mathematical Society, Shorter Notes. pp. 203-204 (1970)

14. Titchmarsh, C.: Introduction to the Theory of Fourier Integrals, 2nd edn. Clarendon Press, Oxford (1948) 
15. Ricci, F.: Hardy Spaces in One Complex Variable, http://homepage.sns.it/fricci/papers/hardy.pdf (2005)

16. Privalov, I.I.: Randeigenschaften analytischer Funktionen. VEB Deutscher Verlag der Wissenschaften, Berlin (1965)

17. Aleman, A.: Introduction to Hardy spaces, www.ctr.maths.lu.sehardy-spaces-lecture-notes2 (2014)

18. Paley, R.E.A.C., Wiener, N.: Fourier transforms in the complex domain. Amer. Math. Soc. Coll. Pub. 19, (1934)

19. Groenewald, G.J., ter Horst, S., Jaftha, J., Ran, A.C.M.: A Toeplitz-like operator with rational symbol having poles on the unit circle I: Fredholm properties, II: The Spectrum, III: The Adjoint, Operator Theory: Advances and Applications 271, 239 - 268 (2018), Operator Theory: Advances and Applications 272, 133 -154 (2019), Integr. Equ. Oper. Theory 91:43 (2019)

20. Hewitt, E., Stromberg, K.: Real and Abstract Analysis. Springer, New York (1969)

21. Vukotić, D.: Analytic Toeplitz operators on the hardy space $H^{p}$ : a Survey. Bull. Belg. Math. Soc. 10, 101-113 (2003)

22. Duduchava, R.: Integral Equations with Fixed Singularities. Teubner, Leipzig (1979)

23. Carleman, T.: L'integrale de Fourier et questions qui s'y rattachent. Almquist Wiksells, Uppsala (1944)

24. Castrigiano, D.P.L.: Spectral Representation of the Wiener-Hopf Operator for the sinc Kernel and some Related Operators, arXiv:2002.09235v1 [math.FA] (2020)

25. Schechter, M.: Basic theory of fredholm operators. Annali della Scuola Normale Superiore di Pisa Classe di Scienze 3e série tome 21(2): 261-280 (1967)

26. Cucker, F., Corbalan, A.G.: An alternate proof of the continuity of the roots of a polynomial. Am. Math. Monthly 96, 342-345 (1989)

27. Rudin, W.: Functional Analysis. TATA McGraw-Hill TMH Edition, New Delhi (1974)

28. Rosenblum, M.: A spectral theory for self-adjoint singular integral operators. Am. J. Math. 88, 314-328 (1966)

29. Putnam, C.R.: Absolute continuity of singular integral operators. Am. J. Math. 91, 453-462 (1969)

30. Pincus, J.D.: Symmetric singular integral operators. Indiana Univ. Math. J. 23, 537-556 (1973)

31. Talenti, G.: Sulle equazioni integrali di Wiener Hopf. Boll. Un. Mat. Ital. (4) 7(1), 18-118 (1973)

32. Widom, H.: Singular integral equations in $L_{p}$. Trans. Amer. Math. Soc. 97, (1960)

33. Koppelman, W., Pincus, J.D.: Spectral representations for finite Hilbert transformations. Math. Zeitschrift 71, 399-407 (1959)

34. Pincus, J.D.: The spectral theory of self-adjoint Wiener-Hopf operators. Bull. Am. Math. Soc. 72, 882-887 (1966)

35. Erdelyi, A., et al.: Higher Transcendental Functions, vol. 2. McGraw-Hill, New York (1953)

36. Gel'fand, I.M., Shilov, G.E.: Generalized Functions, vol. I. Academic Press, New York (1964)

Publisher's Note Springer Nature remains neutral with regard to jurisdictional claims in published maps and institutional affiliations. 